\title{
1 Fitness of breeders in social Damaraland mole-rats is 2 independent of group size
}

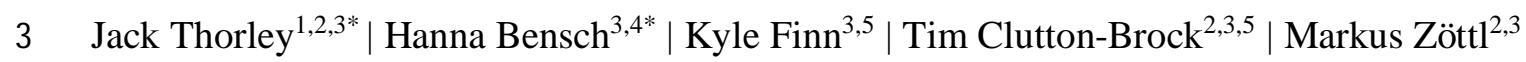

$4 \quad *$ Shared first authorship

$5 \quad{ }^{1}$ Department of Earth, Ocean and Ecological Sciences, University of Liverpool, UK

$6 \quad{ }^{2}$ Department of Zoology, University of Cambridge, Cambridge, UK

$7 \quad{ }^{3}$ Kalahari Research Centre, Kuruman River Reserve, Van Zylsrus, South Africa

$8{ }^{4}$ EEMiS, Department of Biology and Environmental Science, Linnaeus University, Kalmar,

9 Sweden

$10{ }^{5}$ Mammal Research Institute, Department of Zoology and Entomology, University of Pretoria, 110028 Pretoria, South Africa.

12 Corresponding author: Jack Thorley (jack.thorley@liverpool.ac.uk) 
14 Damaraland mole-rats (Fukomys damarensis) are usually viewed as an obligatorily group living

15 eusocial species in which successful reproduction is dependent on reproductive altruism of closely

16 related group members. However, the reproductive ecology of social mole-rats in their natural

17 environment remains poorly understood and it is unclear to what extent successful reproduction is

18 dependent on assistance from other group members. Using data from a 7-year field study of marked

19 individuals, we show that, after dispersal from their natal group, individuals typically settled alone

20 in new burrow systems where they enjoyed high survival rates, and often remained in good body

21 condition for several years before finding a mate. Unlike most other eusocial or singular

22 cooperative breeders, we found that Damaraland mole-rats reproduced successfully in pairs without

23 helpers and experimentally formed pairs had the same reproductive success as larger established

24 groups. Overall there was only a weak increase in reproductive success with increasing group size

25 and no effect of group size on adult survival rates across the population. Juveniles in large groups

26 grew faster early in life but their growth rates declined subsequently so that they eventually

27 plateaued at a lower maximum body mass than juveniles from small groups. Taken together, our

28 data suggest that the fitness benefits of group living to breeders are small and we suggest that

29 extended philopatry in Damaraland mole-rats has evolved because of the high costs and constraints

30 of dispersal rather than because of strong indirect benefits accrued through cooperative behaviour.

32 Keywords: sociality, cooperative breeding, inclusive fitness, philopatry, family living,

33 Bathyergidae 
37 often referred to as the only eusocial mammals on the grounds that there is a single breeding

38 female, that multiple generations overlap, that non-breeders may be allocated to discrete,

39 specialised castes, and that some individuals remain as members of small-bodied worker castes

40 for their entire lives (Jarvis, 1981; Jarvis et al., 1994; Burda et al., 2000; Scantlebury et al.,

41 2006; Faulkes \& Bennett, 2021). It has also been suggested that naked mole-rat groups include

42 a specialised disperser morph similar to the alates of the termites that promotes dispersal and

43 outbreeding (O'Riain, Jarvis \& Faulkes, 1996; Braude, 2000; Toor et al., 2020), and that

44 breeders and non-breeders in both species are morphologically different from one another

45 (O'Riain et al., 2000; Young \& Bennett, 2010; Thorley et al., 2018a). Studies of other social

46 mole-rats of the family Bathyergidae (including Common (Cryptomys hottentotus), Ansell's

47 (Fukomys anselli) and giant mole-rats (Fukomys mechowi)) have shown that groups in these

48 species are generally smaller than those seen in naked and Damaraland mole-rats, but also

49 emphasize strong structural similarities, being comprised of a nuclear family with a single

50 breeding female and limited out-of-group paternity (Bennett \& Faulkes, 2000; Spinks, Bennett

51 \& Jarvis, 2000; Bishop et al., 2004; Patzenhauerová et al., 2013; Caspar, Müller \& Begall,

52 2021; Caspar, Burda \& Begall, 2001). Although there is some uncertainty about how to

53 categorise the various mole-rat species according to their social organisation (Buffenstein et

$54 a l ., 2021$ ), most reviews consider both Damaraland and naked mole-rats as eusocial (Jarvis et

55 al., 1994; Faulkes \& Bennett, 2016, 2021), or all social mole-rats as eusocial (Burda et al.,

56 2000; Braude et al., 2021).

57 Though social mole-rats do share some important characteristics with eusocial and

58 singular cooperatively breeding species, their societies also show some important differences.

59 In groups of social mole-rats, the principal cooperative activity of non-breeding individuals is 
60 to maintain the extensive network of tunnels that provide access to underground plant tubers

61 and to bring food to food stores that are accessible to all group members (Brett, 1991; Bennett

$62 \&$ Faulkes, 2000). This extensive involvement of many non-breeders in cooperative foraging

63 has led to the suggestion that there are strong positive effects of group size on breeding success

64 and survival in groups of social mole-rats, particularly in arid environments (Jarvis et al., 1994;

65 Jarvis, Bennett \& Spinks, 1998). However, direct alloparental care by non-breeders is limited

66 to rare occasions when young offspring wander out of the nest and are retrieved by helpers

67 (Zöttl et al., 2016b, 2018), and unlike in most cooperative breeders (Solomon \& French, 1997;

68 Koenig \& Dickinson, 2016) non-breeders do not provision offspring or the breeding female

69 directly (Bennett \& Faulkes, 2000; Zöttl et al., 2016b). Experiments in captivity have also

70 indicated that territory defence against conspecific intruders in Damaraland mole-rats is

71 predominantly carried out by the breeding individuals (Cooney, 2002), which also contrasts

72 with many other cooperative societies where most group members participate in group defence

73 (Radford \& du Plessis, 2004; Mares, Young \& Clutton-Brock, 2012; Cassidy et al., 2015).

74 Together these studies raise the question of whether most or all of the activities of non-breeders

75 should be regarded as selfish and mutually beneficial to all group members rather than as

76 altruistic behaviours. Currently, there is little evidence of clear, altruistic behaviour of non-

77 breeders directed towards collateral kin and it remains unclear whether non-breeding helpers

78 in mole-rats enhance breeding success or survival of the breeding individuals.

79 To assess to what extent the societies of social mole-rats share characteristics of 80 eusocial breeders and to what extent they resemble those of group living species without 81 substantial altruistic behaviour we need to understand the fitness consequences of group living 82 and cooperative behaviour in natural populations of mole-rats. Longitudinal studies of wild 83 mole-rat populations are particularly uncommon (Faulkes \& Bennett, 2021), but one rare study 84 that explored the effects of group size on various demographic parameters was conducted on a 
85 Damaraland mole-rat population in central Namibia, where Young et al. (2015) found that 86 larger groups recruited more offspring. At the same time, juvenile survival was largely 87 unaffected by group size, and juvenile growth rate was suppressed in large groups; possibly 88 due to competition between non-breeders, and perhaps implying that large groups may also 89 impose costs on the development of offspring (Young et al., 2015). Breeding females have also 90 been shown to have lower workloads than breeders in a separate population of Damaraland 91 mole-rats (Francioli et al., 2020), and in captivity, the workload of breeding females was also 92 reduced in larger groups (Houslay et al., 2020), alluding to helper effects in this setting. 93 However, it is still unclear whether single individuals and small groups in natural populations 94 of social mole-rats experience high mortality, whether nascent groups consisting only of a pair 95 habitually fail with reproduction, whether the presence of non-breeders increases breeder 96 survival and whether group size affects the growth trajectories of juveniles. Different 97 populations of mole-rats will also vary in the environmental conditions they experience and in 98 their demography and this may in turn affect the expression of social traits and the effects of 99 group size at both temporal and spatial scales (Spinks et al., 2000; Finn et al., 2018); as seen 100 for many other social mammals (Silk, 2007; Schradin, König \& Pillay, 2010; Ebensperger, 101 Rivera \& Hayes, 2012; Clutton-Brock, 2016). For any given species the contributions of group 102 members to the various components of fitness cannot therefore be assumed to be consistent 103 across all populations.

In this study we investigate the effects of non-breeders on fitness correlates in a wild 105 population of Damaraland mole-rats located in the southern Kalahari Desert. We first ask 106 whether individuals living in groups experience higher survivorship than individuals that have 107 dispersed and settled solitarily. Second, by experimentally creating breeding pairs in the field, 108 we examine whether the reproductive output of breeders lacking non-breeding group members 109 is lower than that of established groups which have access to a potential workforce. Lastly, we 
110 leverage demographic information collected across 7 years of field study to analyse the

111 associations of group size with (i) reproductive success, (ii) adult survival, and (iii) growth of

112 recruited offspring, testing the prediction that large group size generates reproductive and 113 survival benefits and enhances growth among young individuals.

River Reserve ("Kuruman"), and the Lonely farm ("Lonely", Figures S1 \& S2). The two locations form a continuous population but because of the time intensive nature of mole-rat trapping they tended to be trapped in discrete periods (Figures S3 \& S4), hereafter referred to as “trapping windows". For logistical reasons, trapping stopped at Lonely in July 2017. the laboratory where they were sexes, weighed to the nearest gram and measured for various 
134 natural tunnel systems in the laboratory and provided with food, nesting material and sand. The

135 complete group was assumed to have been captured after an absence of activity at the trap sites

136 for $24 \mathrm{hrs}$, after which point the animals were returned to their tunnel system.

137 Between September 2013 and May 2020, 752 unique individuals (368 females, 384

138 males $)$ were trapped at the two sites $(\mathrm{N}=1941$ individual captures $)$. The mean recapture-rate

139 of individuals across successive trapping windows was $73.1 \%$ at Kuruman and $54.8 \%$ at Lonely

140 (Table S1). In total, analyses used data from 328 group captures that were carried out at 84

141 groups with mean of $4.0(1 \mathrm{SD}=3.07)$ captures per group (Figure S2). Groups were defined as

142 individuals repeatedly found at the same trapping location, and in the majority of recapture

143 events the same breeding female remained at a group for their duration. Thus, as long as the

144 majority of individuals trapped within the same area were known to originate from the same

145 group, the group continued to be defined as such. On several occasions a new group had moved

146 into the tunnel system previously occupied by another group. The initial group were then either

147 found elsewhere or never recaptured and likely extinct. Single individuals, either new unknown

148 individuals or known individuals that had dispersed from an established group were assigned

149 their own unique group ID, which was retained if they were later joined by an immigrant

150 partner and started breeding in the same area.

151 In $15.2 \%$ of group captures, continued activity at trapping sites indicated that the 152 complete group had not been captured by the end of the week of trapping. The decision to 153 include or exclude these incomplete captures depended on the analysis: group-level analyses 154 excluded incomplete captures whereas individual-level analyses included them (a summary of 155 the data and models used for the various analyses is provided in Table S2.). Analyses were 156 conducted in R version 3.6.3 (R Core Team, 2021), and all code is provided online (Github, to 157 be made public on acceptance). 


\section{b) Status-related survivorship among females}

To estimate the survivorship of females in different states we fitted a multi-state

Markov model. Such models are typically used in a medical setting for 'panel data', where a

continuously observed process - like disease progression - is measured only at discrete time

points - when people choose to visit the hospital. The timing of transitions between states can

then be estimated indirectly under the assumption that the next state in a sequence of states

depends only on the current state, and not on the history of transitions or on the time spent in

the prior state (Jackson, 2011). Our longitudinal data bears a panel-like structure, with

individuals and groups being periodically captured (or not) in different life history stages or

'states'. The model then estimates the probability that individual mole-rats transition between

different states. Here, we present information on survival probability from each state.

Information on individual capture histories was used to assign individuals to one of four

states relative to the time since their first capture: $i$ - non-breeder in their natal group, ii- single

individual, iii- breeding individual out of their natal group, iv-disappeared/dead (Figure S5).

172 For model fitting purposes, in the small number of cases where females inherited a breeding

173 position within their natal group $(n=7)$, these females were categorised in the same state as

174 females that acquired a breeding position out of their natal group (iii). To define state $i v$

175 (disappeared/died), we incorporated information from the trapping windows at "Kuruman" and

176 "Lonely". If an individual had not been recaptured for at least two consecutive trapping

177 windows, it was assumed to have dispersed or died at some point between its last live capture

178 and the start of the subsequent trapping window, and given an extra row of data reflecting this;

179 the model is then parameterised so that the timing of disappearance or death is not exact.

180 Similarly, if individuals were captured within the last trapping window at each location, we

181 assumed that they were still alive at the end of that trapping window (i.e. the end of the study).

182 They were then given an extra row of data reflecting this assumption and were censored at this 
183 point, which in the most extreme case assumes that individuals remained in their last captured

184 state for a further 80 days (Figure S5). To explore whether group size affected the likelihood

185 that non-breeding females or breeding females died/disappeared, we fitted an additional model

186 that included a group size covariate for each of these transitions. The multi-state models were

187 fitted in the msm package (Jackson, 2011; Figure S6 for multi-state diagram). When reporting

188 hazard ratios and relative likelihoods from the multi-state models we class cases where the $95 \%$

189 confidence intervals do not overlap one as indicating a biologically important effect, and

190 otherwise emphasize effect sizes.

\section{c) Within-group recruitment}

Two approaches were used to investigate the role of group size on within-group

recruitment. In the first, recruitment was analysed longitudinally across the duration of the

study. In the second, new groups were experimentally created in the wild through the

introduction of unfamiliar adult males to solitary females. The recruitment rate of newly

created pairs was then compared to that of established groups that were captured and recaptured

within the same time period. The benefit of the second approach is that it allows for a direct

test of whether groups in the early stages of group formation were less productive than

established groups. Any individuals caught within one year that were lighter than $100 \mathrm{~g}$ (males)

represent out-of-group immigrants.

a group was captured and then recaptured within a 100 to 365-day time period; where a resident

breeding female was present; and where at least one large male was retained across the two 
207 dataset of 78 group-level capture-recapture events that took place in 33 groups with a mean

208 trapping interval of 214.2 days $(1 \mathrm{SD}=52.11)$. The number of recruits was modelled using a

209 generalised linear mixed effects model with Poisson error in the $g \operatorname{lmm} T M B$ package (Brooks,

210 Mollie et al., 2017). Fixed effects of group size at first capture, the mass of the breeding female

211 at the capture, and rainfall were included. A single random effect of group identity was

212 included, and the logged time interval between capture and recapture was included as an offset

213 term. Rainfall was calculated as the geometric mean monthly rainfall in the year preceding the

214 first capture as this provided the best fit to the data (lowest AIC score) when compared to

215 models fitted to the total rainfall, or the arithmetic mean rainfall. After standardising relative

216 to the trapping duration, the mean 6-monthly recruitment to groups was 2.12 individuals (1SD

$217=1.82$ ). Continuous variables were z-score transformed prior to model fitting.

218 For the experimental approach, 8 solitary females were captured between $16^{\text {th }}$ April

2192016 and 29 2 th May 2016. Solitary females were brought back into the laboratory and paired

220 with wild males captured concurrently from intact breeding groups. When pairing, males and

221 females were first isolated in their own temporary tunnel system and exposed to the odour of

222 their prospective partner for 24 hours. After this time period, the male was introduced to the

223 female for 24 hours in her lab tunnel system, before they were returned together to the female's

224 tunnel system in the wild. All pairings used large adult males originating from groups $>3 \mathrm{~km}$

225 from the focal solitary female, so it is highly unlikely that pairings were conducted between

226 close relatives and/or familiar individuals. The recruitment rate of new pairs was compared to

227 that of established groups captured over the same time period using a Welch's t-test after first

228 standardising recruitment rate to a yearly measure. Groups where a male was removed were

229 not included in the established groups treatment in case the male was a breeder in his original

230 group, which would necessarily reduce the rate of recruitment. The mean trapping interval did 
231 not differ between new pairs (249.89 days \pm 94.34$)$ and established groups (306.88 days \pm

232 64.39; Welch's t-test: $\mathrm{t}=-1.47, \mathrm{df}=14.14, \mathrm{p}=0.16)$.

As the age of wild-caught individuals was unknown, we modelled growth using interval equations that estimated the change in body mass and upper incisor width of individuals across

Damaraland mole-rats and are the main apparatus of digging (Young \& Bennett, 2010). Incisor

were taken in duplicate by two observers and the mean of these two measurements was used.

Previous studies show that the shape of growth in captive mole-rats is concave and can be

approximated by a monomolecular curve (Thorley, 2018). To allow for a similar shape of

the nlme package (Pinheiro et al., 2019). The body mass or incisor width of an individual at each recapture was estimated as:

$$
S 2_{i}=A-\left(A-S 1_{i}\right) e^{-k \cdot D_{i}}+\varepsilon_{i}
$$

where $\mathrm{S} 1_{i}$ and $\mathrm{S} 2_{i}$ are the size at capture and recapture, respectively, for individual $i$. A

248 is the estimated population-level asymptotic size, $k$ is the growth rate constant, $D_{i}$ is the time

249 difference between capture and recapture (hereafter the 'trapping interval'). $\varepsilon_{\mathrm{i}}$ is the normally

250 distributed error with mean 0 and variance $\sigma^{2}$.

251 Separate models were fitted to males and females for each size metric, using all

252 information from individuals that were recaptured at least once, and where the recapture 253 interval fell between 90 and 365 days. The female body mass dataset comprised 381 "repeat 254 capture" events $(n=193$ females, mean/female $=1.96,1 \mathrm{SD}=1.21)$ and excluded any weight 
255 data from females once they were known to be a breeder, thus removing any artefact of status

256 or pregnancy. The male body mass dataset consisted of $n=456$ repeat capture events $(n=214$

257 males, mean $/$ male $=2.13,1 \mathrm{SD}=1.54)$. The female and male incisor width datasets consisted

258 of 328 and 381 repeat capture events respectively $(n=193$ females, mean/female $=1.82,1 \mathrm{SD}$

$259=1.16 ; \mathrm{n}=198$ males, mean $/$ male $=1.92,1 \mathrm{SD}=1.33)$. In each model a random effect of

260 individual identity was specified at the level of the asymptote and the growth rate constant, and

261 to aid convergence, random effects were modelled as uncorrelated. Likelihood ratio tests

262 indicated that the inclusion of a random effect of group identity was not needed.

263 The initial growth model was extended by incorporating a standardised group size term:

$$
S 2_{i}=\left(A+A_{G S} \cdot G S\right)-\left(\left(A+A_{G S} \cdot G S\right)-S 1_{i}\right) e^{-\left(k+k_{G S} \cdot G S\right) \cdot D_{i}}+\varepsilon_{i}
$$

Here, $A_{\mathrm{GS}}$ and $k_{\mathrm{GS}}$ estimate the change in asymptotic mass and growth rate constant with changing group size, GS. Group size was taken as the group size on initial capture, which was

267 highly correlated with recapture group size $(\mathrm{r}=0.78, \mathrm{df}=835, \mathrm{p}<0.001)$. As the results were qualitatively unaffected by excluding incomplete captures, results are presented for all 


\section{RESULTS}

\section{a) Population structure and distribution of group sizes}

The mean group size across all captures was 5.91 individuals $(1 \mathrm{SD}=5.61$, $\max =26$,

274 Figure 1). Of the 84 unique groups that were captured, 49 were of a single individual on the 275 first capture, 94\% of which were females $(n=46)$. Excluding this large number of single 276 individuals, the mean group size was 8.67 individuals $(1 \mathrm{SD}=5.30)$, which can be taken to 277 represent the average size of a breeding unit in the population.

278 Across the duration of the study only one reproductively active 'breeding' female was 279 present at a group at any given time.

\section{b) Status-related survivorship among females}

Single females generally exhibited high annual survival rates and disappeared from the

population at intermediate rates between those of breeders and those of non-breeders (Figure

2, Table S3); their survival was higher (79.3\%) than that of in-group non-breeders (64.2\%) and

tended to be lower than that of breeders $(86.7 \%)$. Thus, in-group non-breeders were 1.91 times

more likely to disappear than single females (95\% CI [1.07,3.43]), and 3.28 times more likely

to disappear than breeding females (95\% CI [1.84,5.82]). The rate of disappearance of single

287 females was higher than that of breeding females, with the former being 1.71 times more likely

to disappear than the latter, though this effect was not significant (95\% CI [0.78,3.77], Figure

2c). Group size did not appear to affect the likelihood that non-breeding females (hazard ratio

$=1.003,95 \% \mathrm{CI}[0.973,1.033])$ or breeding females (hazard ratio $=1.058,95 \% \mathrm{CI}$ 
294 breeders (Figure 3), suggesting that solitary living does not affect the ability of individuals to 295 acquire food and maintain mass (condition analyses outlined in Supplementary Material).

\section{c) Within-group recruitment}

The rate of recruitment was largely independent of group size. Experimentally created

pairs recruited a comparable number of offspring into their group as established groups that

were captured and recaptured over the same time period (Figure 4a; Welch's t-test, $t=0.253$,

$\mathrm{df}=14.02, \mathrm{p}=0.80$; Figure $\mathrm{S} 7$ details capture history after pairing). The recruitment rate in

experimental and control groups over the experimental period was comparable to the total

recruitment rate over the whole study period (Figure 4a), and environmental conditions across

the experimental period were typical of those seen in the Kalahari over a longer time span

304 (Figure S8).

Across the duration of the study, group size was associated with a slight increase in withingroup recruitment rate $(0.216 \pm 0.106, \mathrm{z}=2.04, \mathrm{p}=0.042$, Table $\mathrm{S} 4$, Figure $4 \mathrm{~b})$. Changing the group size term to reflect the mean group size across the capture and recapture event did not

qualitatively affect the result. Increased rainfall in the year prior to first trapping trended towards having a positive effect on recruitment (geometric mean monthly rainfall: $0.159 \pm$ $0.086, \mathrm{z}=1.85, \mathrm{p}=0.065)$, but the body mass of the breeding female did not $(-0.090 \pm 0.089$,

$\mathrm{z}=-1.02, \mathrm{p}=0.31$ ). The inclusion of an interaction between rainfall and group size suggested weather conditions $(0.108 \pm 0.080, \mathrm{z}=1.36, \mathrm{p}=0.17)$. 
317 larger groups and their members ultimately went on to attain a lower asymptotic body mass

318 (NLMM: male $A_{G S}=-8.10 \pm 2.02, \mathrm{p}<0.001$; female $\left.A_{G S}=-9.42 \pm 1.47, \mathrm{p}<0.001\right)$ (see Figures

319 S9-10, Supplementary Material for further treatment). Increases in group size were also 320 associated with reductions in asymptotic skeletal size in both sexes, though the effect was only 321 supported in females (NLMM: male $A_{G S}=-0.047 \pm 0.036, \mathrm{p}=0.19$; female $A_{G S}=-0.113 \pm$ $3220.027, \mathrm{p}<0.001$, Table S7). In contrast to the body mass models, there was no support for a relationship between group size and the growth rate constant in either sex (Table S7).

\section{DISCUSSION}

The idea that there are strong benefits of group-living to breeders is implicit in many discussions surrounding the evolution of sociality in the African mole-rats (Jarvis et al., 1994; Faulkes \& Bennett, 2021). However, up until now only a single study of social mole-rats has explored the fitness consequences of group size in a wild population. In this previous study of Damaraland mole-rats, Young et al. (2015) found that reproductive success was higher in large groups, that survival was independent of group size, and that growth rates of juveniles were reduced in larger groups. In captivity, Houslay et al. (2020) also demonstrated that breeding females in larger groups worked less and showed higher fecundity, though juveniles in this and other captive populations grew more slowly in large groups despite being fed ad libitum (Bennett \& Navarro, 1997; Zöttl et al., 2016a).

Contrary to the long held belief that group size has strong positive effects on breeder

336 fitness in social mole-rats, our detailed demographic analysis of Damaraland mole-rats 337 demonstrates that for our study population, group size effects are often weak and can be offset 338 by costs of breeding in large groups. In other social species, competition within groups can reduce growth (Buston 2003; Heg et al. 2004) and it is possible that similar effects of competition also reduce fitness in mole-rats. Despite the many possible benefits of large group 
341 size, the weakness or absence of positive associations between group size and fitness correlates

342 overall indicates that the inclusive fitness benefits generated by non-breeders are probably low.

343 We suggest that ecological constraints on dispersal should be considered the principal driver

344 of group-living in the African mole-rats and our data provides several lines of evidence that 345 support this suggestion.

346 A significant proportion of individuals in our study population are females that have 347 dispersed and settled alone in a burrow system. We find that these solitary individuals display 348 high survivorship and good body condition, despite being forced to forage alone and lacking 349 access to other putative benefits of group living, such as the benefits of group defence, or social 350 thermoregulation. Once joined by a male, these nascent pairs often begin breeding immediately 351 and produce pups at a rate which is comparable to that of established groups. This supports the 352 more general picture to emerge from our data that increases in group size are associated with 353 minimal increases in the reproductive output of breeders. We also show that pups born in small 354 groups initially grow more slowly than pups born in large groups but that their subsequent asymptotic mass is higher than that of individuals in larger groups. These are uncommon 356 patterns among cooperatively breeding vertebrates - to which mole-rats are often compared 357 where the effects of group size on fitness are often strongly positive (Solomon \& French, 1997; 358 Russell, 2004; Ebensperger et al., 2012). This is particularly true of singular cooperative 359 breeders like African wild dogs (Lycaon pictus), meerkats (Suricata suricatta) and dwarf 360 mongooses (Helogale parvula), where a breeding pair is responsible for the majority of group361 level reproduction and where pairs of individuals usually fail to reproduce successfully without 362 the direct actions of helpers in rearing pups (Rood, 1990; Creel \& Creel, 2002; Groenewoud \& 363 Clutton-Brock, 2021). We of course recognise that large group sizes can also have negative 364 impacts in these species (e.g. Creel \& Creel, 2015), but to find only weak or absent effects 
365 across several parameters as we do in our study is unusual, and challenges the idea that

366 Damaraland mole-rat breeders are reliant on assistance from non-breeders.

367 Instead of being maintained by selection for reproductive cooperation, group-living in

368 mole-rats may be largely due to ecological constraints on dispersal. Such constraints appear to

369 have been important in the evolution of group-living in rodents generally (Solomon, 2003;

370 Firman et al., 2020), and while often also depending on phylogeny and other aspects of life

371 history (Ebensperger \& Blumstein, 2006; Smorkatcheva \& Lukhtanov, 2014; Sobrero et al.,

372 2014), the low recapture rate of non-breeding individuals in our study testifies to the high risks

373 of dispersal (which is often overground) for Damaraland mole-rats (see also Hazell et al.,

374 2000). This likely explains why dispersal in this species is concentrated around intermittent

375 periods of rainfall - when the soil is soft enough to facilitate burrow digging (Lovegrove, 1989;

376 Jarvis et al., 1994; Torrents Ticó et al., 2018a)- and why dispersal success is strongly associated

377 with individual quality, such that fast-growing individuals that reach a high pre-departure body

378 mass are most likely to establish independent breeding territories (Torrents-Ticó et al., 2018b).

379 As a result, there is likely to be strong selection on non-breeders to delay dispersal and remain

380 in their natal group until their chances of dispersing successfully are greatest (Jarvis et al.,

381 1998; Bennett \& Faulkes, 2000). Breeders, in turn, may tolerate non-breeders as a form of

382 extended parental care even if their retained offspring provide little to no fitness benefits or

383 advantage their future reproduction. This argument implies that maternal tolerance towards

384 offspring beyond their independence may have been a key factor facilitating group-living in

385 the social mole-rats. Observations of parental behaviour in captive mole-rats support this idea.

386 In solitary species, researchers have noted frequent aggression from mothers towards offspring

387 and have therefore stated that natal dispersal is often driven by eviction in these species

388 (Bennett \& Faulkes, 2000; Šumbera, Burda \& Chitaukali, 2003). By contrast, our observations

389 of captive Damaraland mole-rats indicate that interactions between mothers and offspring are 
390 rarely aggressive, and that evictions of retained offspring from intact family groups are

391 uncommon. It therefore seems likely that most dispersal events from wild mole-rat groups are 392 voluntary.

393 With respect to philopatry of non-breeders and cooperative behaviour within groups, 394 our results suggest that Damaraland mole-rat societies resemble those of some group-living 395 birds and other social rodents that live in families but where helper effects are minimal or 396 absent (Ekman, 2006; Drobniak et al., 2015). Siberian jays (Perisoreus infaustus) for example, 397 live in groups where recruits remain in their parents' territory, do not breed themselves and 398 play little role in raising their parents subsequent offspring (Ekman \& Griesser, 2016). Like in 399 mole-rats, non-breeders in these bird groups delay dispersal due to ecological constraints and 400 because early departure from the group greatly diminishes the chances of successfully 401 establishing a breeding territory (Ekman, Bylin \& Tegelström, 1999; Torrents-Ticó et al., 402 2018b). In their natal group, philopatric offspring participate in group-level collective actions 403 such as coordinated territory defence and - in the case of mole-rats - in communal foraging, 404 though do not directly provision subsequent offspring, and there are likely fitness benefits for 405 all group members that are generated by this form of mutually beneficial cooperation. 406 Similarly, in other social rodents, such as marmots (Marmota sp.), and beavers (Castor sp.), 407 group members delay their dispersal and engage in shared, mutually beneficial activities such 408 as nest building, burrow/den construction, and huddling for thermoregulation (Blumstein \& 409 Armitage, 1999; Busher, 2007; Mayer, Zedrosser \& Rosell, 2017). However, the presence of 410 older offspring may also impose costs on breeders and subsequent offspring through enhanced 411 resource competition and intrasexual competition and in Damaraland mole-rats breeders 412 probably tolerate these costs because the benefits either outweigh the costs, so that the 413 prolonged presence of non-breeders comes to a net zero outcome, or because they are prepared 414 to tolerate some costs to their future reproduction as a form of extended parental care. 
415 While our study found little support for clear group size effects on fitness, it remains

416 possible that some benefits of group size only become apparent under extreme environmental

417 conditions, such as during extended droughts (Jarvis et al., 1998; Bourne et al., 2020; Capilla-

418 Lasheras et al., 2021; Groenewoud \& Clutton-Brock, 2021), or are masked by counteracting

419 pressures, such as increased competition for food, or elevated parasite burdens (Schmid-

420 Hempel, 2017). There are also other ways in which group-living might benefit individuals

421 which we have not been able to fully investigate in our population thus far. Chief among these

422 is the possibility that larger groups persist for longer than smaller groups (Keynan \& Ridley,

423 2016; Duncan, Manser \& Clutton-Brock, 2021). Our finding that group size has little effect on

424 breeder survival, and that single females enjoy high survivorship, downplays the importance

425 of group size on group persistence generally, but the long potential lifespan of Damaraland

426 mole-rats (more than 10 years: Schmidt, Jarvis \& Bennett, 2013) might mean that particularly

427 long time series on the order of several decades are needed to critically examine group

428 persistence.

429 Overall, our data suggest that the idea that some non-breeders have adopted an 430 alternative life-history strategy of lifetime philopatry similar to workers found in eusocial 431 insects is probably incorrect, because non-breeders appear to gain little inclusive fitness from 432 philopatry. Instead, individuals that fail to acquire a breeding position are probably low-quality 433 individuals that show reduced growth rates and lower investments in cooperative behaviour 434 prior to dispersal (Zöttl et al., 2016b; Thorley et al., 2018b; Torrents-Ticó et al., 2018b). While 435 we acknowledge the similarity of Damaraland mole-rat societies to eusocial and cooperative 436 breeders in terms of reproductive skew and reproductive suppression within groups (Faulkes 437 \& Bennett, 2016, 2021; Braude et al., 2021), our paper highlights that further similarities 438 relating to the positive effects of helpers on breeder reproduction have been overemphasized. 
439 In this latter aspect, Damaraland mole-rats may be more similar to some group-living birds and 440 social rodents where breeders do not rely on the assistance of helpers.

\section{REFERENCES}

Bennett, N.C. \& Faulkes, C.G. (2000). The African Mole-rats: Ecology and Eusociality. Cambridge: Cambridge University Press.

Bennett, N.C. \& Navarro, R. (1997). Differential growth patterns between successive litters of the eusocial Damaraland mole-rat, Cryptomys damarensis, from Namibia. J. Zool. 241, 465-473.

Bishop, J.M., Jarvis, J.U.M., Spinks, A.C., Bennett, N.C. \& O’Ryan, C. (2004). Molecular insight into patterns of colony composition and paternity in the common mole-rat Cryptomys hottentotus hottentotus. Mol. Ecol. 13, 1217-1229.

Blumstein, D.T. \& Armitage, K. (1999). Coperative breeding in marmots. Oikos 84, 369-382.

Bourne, A.R., Cunningham, S.J., Spottiswoode, C.N. \& Ridley, A.R. (2020). Hot droughts compromise interannual survival across all group sizes in a cooperatively breeding bird. Ecol. Lett. 23, 17761788 .

Braude, S., Holtze, S., Begall, S., Brenmoehl, J., Burda, H., Dammann, P., Marmol, D., Gorshkova, E.,
Henning, Y., Hoeflich, A., Höhn, A., Jung, T., Hamo, D., Sahm, A., Shebzukhov, Y., Šumbera,
R., Miwa, S., Vyssokikh, M.Y., Zglinicki, T., Averina, O. \& Hildebrandt, T.B. (2021).

Braude, S., Holtze, S., Begall, S., Brenmoehl, J., Burda, H., Dammann, P., Marmol, D., Gorshkova, E.,
Henning, Y., Hoeflich, A., Höhn, A., Jung, T., Hamo, D., Sahm, A., Shebzukhov, Y., Šumbera,
R., Miwa, S., Vyssokikh, M.Y., Zglinicki, T., Averina, O. \& Hildebrandt, T.B. (2021).

Braude, S. (2000). Dispersal and new colony formation in wild naked mole-rats: evidence against inbreeding as the system of mating. Behav. Ecol. 11, 7-12. Surprisingly long survival of premature conclusions about naked mole-rat biology. Biol. Rev. 96, 376-393.

Brett, R. (1991). The ecology of naked mole-rat colonies: burrowing, food and limiting factors. In The Biology of the Naked Mole Rat: 137-184. Jarvis, J.U.M., Sherman, P.W. \& Alexander, R.D. (Eds.). Princeton, NJ: Princeton University Press.

Brooks, Mollie, E., Kristensen, K., Benthem, Koen, J., V., Magnusson, A., Berg, Casper, W., Nielsen, A., Skaug, Hans, J., Mächler, M. \& Bolker, Benjamin, M. (2017). glmmTMB Balances Speed and Flexibility Among Packages for Zero-inflated Generalized Linear Mixed Modeling. The $R$ Journal. 9, 378-400.

Buffenstein, R., Amoroso, V., Andziak, B., Avdieiev, S., Azpurua, J., Barker, A.J., Bennett, N.C., Brieño-Enríquez, M.A., Bronner, G.N., Coen, C., et al. (2021). The naked truth: a comprehensive clarification and classification of current 'myths' in naked mole-rat biology. Biol. Rev. brv.12791.

Burda, H., Honeycutt, R.L., Begall, S., Locker-Grütjen, O. \& Scharff, A. (2000). Are naked and common mole-rats eusocial and if so, why? Behav. Ecol. Sociobiol. 47, 293-303. 
Busher, P. (2007). Social organisation and monogamy in the beaver. In Rodent Societies: An Ecological and Evolutionary Perspective: 280-290. Wolff, J.O. \& Sherman, P.W. (Eds.). Chigago: Chicago University Press.

Capilla-Lasheras, P., Harrison, X., Wood, E.M., Wilson, A.J. \& Young, A.J. (2021). Altruistic bethedging and the evolution of cooperation in a Kalahari bird. Sci. Adv. 7, 1-11.

Caspar, K.R., Burda, H. \& Begall, S. (2021) Fukomys mechowii (Rodentia: Bathyergidae). Mamm. Species, 53(1011), 145-149.

Cassidy, K.A., MacNulty, D.R., Stahler, D.R., Smith, D.W. \& Mech, L.D. (2015). Group composition effects on aggressive interpack interactions of gray wolves in Yellowstone National Park. Behav. Ecol. 26, 1352-1360.

Clutton-Brock, T. (2016). Mammal Societies. Chichester, UK: Wiley.

Cooney, R. (2002). Colony defense in Damaraland mole-rats, Cryptomys damarensis. Behav. Ecol. 13, $160-162$.

Creel, S. \& Creel, N.M. (2002). The African Wild Dog: Behaviour, Ecology and Evolution. Princeton, NJ: Princeton University Press.

Creel, S. \& Creel, N.M. (2015). Opposing effects of group size on reproduction and survival in African wild dogs. Behav. Ecol. 26, 1414-1422.

Drobniak, S.M., Wagner, G., Mourocq, E. \& Griesser, M. (2015). Family living: An overlooked but pivotal social system to understand the evolution of cooperative breeding. Behav. Ecol. 26, 805811.

Duncan, C., Manser, M.B. \& Clutton-Brock, T. (2021). Decline and fall: The causes of group failure in cooperatively breeding meerkats. Ecol. Evol. 11, 14459-14474.

Ebensperger, L.A. \& Blumstein, D.T. (2006). Sociality in New World hystricognath rodents is linked to predators and burrow digging. Behav. Ecol. 17, 410-418.

Ebensperger, L.A., Rivera, D.S. \& Hayes, L.D. (2012). Direct fitness of group living mammals varies with breeding strategy, climate and fitness estimates. J. Anim. Ecol. 81, 1013-1023.

Ekman, J. (2006). Family Living among Birds. J. Avian Biol. 37, 289-298.

Ekman, J., Bylin, A. \& Tegelström, H. (1999). Increased lifetime reproductive success for Siberian jay (Perisoreus infaustus) males with delayed dispersal. Proc. R. Soc. London. Ser. B Biol. Sci. 266, 911-915.

Ekman, J. \& Griesser, M. (2016). Siberian jays: Delayed dispersal in the absence of cooperative breeding. In Cooperative Breeding in Vertebrates: 6-18. Koenig, W.D. \& Dickinson, J.L. (Eds.).

508 Faulkes, C.G. \& Bennett, N.C. (2016). Damaraland and naked mole-rats: Convergence of social 
evolution. In Cooperative Breeding in Vertebrates: Studies of Ecology, Evolution, and Behavior: 338-253. Koenig, W.D. \& Dickinson, J.L. (Eds.). Cambridge, UK: Cambridge University Press.

Faulkes, C.G. \& Bennett, N.C. (2021). Social Evolution in African Mole-Rats - A Comparative Overview. In The extraordinary biology of the naked mole-rat. Advances in Experimental Medicine and Biology, vol 1319.: 1-33. Buffenstein, R.B., Park, T.J. \& Holmes, M.M. (Eds.). Springer, Cham.

Finn, K.T., Parker, D.M., Bennett, N.C. \& Zöttl, M. (2018). Contrasts in body size and growth suggest that high population density results in faster pace of life in damaraland mole-rats (Fukomys damarensis). Can. J. Zool. 96, 920-927.

Firman, R.C., Rubenstein, D.R., Moran, J.M., Rowe, K.C. \& Buzatto, B.A. (2020). Extreme and variable climatic conditions drive the evolution of sociality in Australian rodents. Curr. Biol. 30, 691-697.e3.

Francioli, Y., Thorley, J., Finn, K., Clutton-Brock, T. \& Zöttl, M. (2020). Breeders are less active foragers than non-breeders in wild Damaraland mole-rats. Biol. Lett. 16, 20200475.

Groenewoud, F. \& Clutton-Brock, T. (2021). Meerkat helpers buffer the detrimental effects of adverse environmental conditions on fecundity, growth and survival. J. Anim. Ecol. 90, 641-652.

Hazell, R.W.A., Bennett, N.C., Jarvis, J.U.M. \& Grif, M. (2000). Adult dispersal in the co-operatively breeding Damaraland mole-rat (Cryptomys damarensis ): a case study from the Waterberg region of Namibia. J. Zool. 252, 19-25.

Houslay, T.M., Vullioud, P., Zöttl, M. \& Clutton-Brock, T.H. (2020). Benefits of cooperation in captive Damaraland mole-rats. Behav. Ecol. 31, 711-718.

Jackson, C.H. (2011). Multi-state models for panel data: the msm package for R. J. Stat. Softw. 28, 129.

Jarvis, J.U.M. (1981). Eusociality in a mammal: Cooperative breeding in naked mole-rat colonies. Science (80-. ). 212, 571-573.

Jarvis, J.U.M., Bennett, N.C. \& Spinks, A.C. (1998). Food availability and foraging by wild colonies of Damaraland mole-rats (Cryptomys damarensis): implications for sociality. Oecologia 113, 290-298.

Jarvis, J.U.M., O'Riain, M.J., Bennett, N.C. \& Sherman, P.W. (1994). Mammalian eusociality: a family affair. Trends Ecol. Evol. 9, 47-51.

Keynan, O. \& Ridley, A.R. (2016). Component, group and demographic Allee effects in a cooperatively breeding bird species, the Arabian babbler (Turdoides squamiceps). Oecologia 182, 153-161.

Koenig, W.D. \& Dickinson, J.L. (2016). Cooperative breeding in vertebrates. Cambridge University Press.

Lovegrove, B.G. (1989). Costs of burrowing for social mole rats (Bathygerdiae) Cryptomys damarensis

545 Mares, R., Young, A.J. \& Clutton-Brock, T.H. (2012). Individual contributions to territory defence in 

3995.

Mayer, M., Zedrosser, A. \& Rosell, F. (2017). When to leave: the timing of natal dispersal in a large, monogamous rodent, the Eurasian beaver. Anim. Behav. 123, 375-382.

O’Riain, M.J., Jarvis, J.U.M., Alexander, R., Buffenstein, R. \& Peeters, C. (2000). Morphological castes in a vertebrate. Proc. Natl. Acad. Sci. 97, 13194-13197.

O’Riain, M.J., Jarvis, J.U.M. \& Faulkes, C.G. (1996). A dispersive morph in the naked mole-rat. Nature 380, 619-621.

Patzenhauerová, H., Šklíba, J., Bryja, J. \& Šumbera, R. (2013). Parentage analysis of Ansell's mole-rat family groups indicates a high reproductive skew despite relatively relaxed ecological constraints on dispersal. Mol. Ecol. 22, 4988-5000.

Pinheiro, J., Bates, D., Debroy, S., Sarkar, D. \& Team, R.C. (2019). nlme: Linear and Nonlinear Mixed Effects Models package version 3.1.

R Core Team. (2021). R: A language and environment for statistical computing. Vienna, Austria.

Radford, A.N. \& du Plessis, M.A. (2004). Territorial vocal rallying in the green woodhoopoe: factors affecting contest length and outcome. Anim. Behav. 68, 803-810.

Rood, J.P. (1990). Group size, survival, reproduction, and routes to breeding in dwarf mongooses. Anim. Behav. 39, 566-572.

Russell, A.F. (2004). Mammals: comparisons and contrasts. In Ecology and evolution of cooperative breeding in birds: 210-227. Koenig, W.D. \& Dickinson, J.. (Eds.). Cambridge: Cambridge University Press.

Scantlebury, M., Speakman, J.R., Oosthuizen, M.K., Roper, T.J. \& Bennett, N.C. (2006). Energetics reveals physiologically distinct castes in a eusocial mammal. Nature 440, 795-797.

Schmid-Hempel, P. (2017). Parasites and Their Social Hosts. Trends Parasitol. 33, 453-462.

Schmidt, C.M., Jarvis, J.U.M. \& Bennett, N.C. (2013). The long-lived queen: reproduction and longevity in female eusocial Damaraland mole-rats. African Zool. 48, 193-196.

Schradin, C., König, B. \& Pillay, N. (2010). Reproductive competition favours solitary living while ecological constraints impose group-living in African striped mice. J. Anim. Ecol. 79, 515-521.

Silk, J.B. (2007). The adaptive value of sociality in mammalian groups. Philos. Trans. R. Soc. B Biol. Sci. 362, 539-559.

Smorkatcheva, A.V. \& Lukhtanov, V.A. (2014). Evolutionary association between subterranean lifestyle and female sociality in rodents. Mamm. Biol. 79, 101-109.

Sobrero, R., Inostroza-Michael, O., Hernández, C.E. \& Ebensperger, L.A. (2014). Phylogeny modulates the effects of ecological conditions on group living across hystricognath rodents. Anim. Behav. 
Solomon, N.G. \& French, J.A. (1997). Cooperative Breeding in Mammals. Cambridge: Cambridge University Press.

Spinks, A., Bennett, N. \& Jarvis, J. (2000). A comparison of the ecology of two populations of the common mole-rat, Cryptomys hottentotus hottentotus : the effect of aridity on food, foraging and body mass. Oecologia 125, 341-349.

Šumbera, R., Burda, H. \& Chitaukali, W.N. (2003). Reproductive biology of a solitary subterranean Bathyergid Rodent, The Silvery Mole-rat (Heliophobius Argentocinereus). J. Mammal. 84, 278287.

Thorley, J. (2018). The shape of growth in Damaraland mole-rats: sex and social effects. The life history of Damaraland mole-rats Fukomys damarensis: growth, ageing and behaviour (PhD Thesis): 5779. University of Cambridge.

Thorley, J. \& Clutton-Brock, T.H. (2019). A unified-models analysis of the development of sexual size dimorphism in Damaraland mole-rats, Fukomys damarensis. J. Mammal. 100, 1374-1386.

Thorley, J., Katlein, N., Goddard, K., Zöttl, M. \& Clutton-Brock, T. (2018a). Reproduction triggers adaptive increases in body size in female mole-rats. Proc. R. Soc. B Biol. Sci. 285, 20180897.

Thorley, J., Mendonça, R., Vullioud, P., Torrents-Ticó, M., Zöttl, M., Gaynor, D. \& Clutton-Brock, T. (2018b). No task specialization among helpers in Damaraland mole-rats. Anim. Behav. 143, 9-24.

Toor, I., Edwards, P.D., Kaka, N., Whitney, R., Ziolkowski, J., Monks, D.A. \& Holmes, M.M. (2020). Aggression and motivation to disperse in eusocial naked mole-rats, Heterocephalus glaber. Anim. Behav. 168, 45-58.

Torrents Ticó, M., Bennett, N.C., Jarvis, J.U.M. \& Zöttl, M. (2018a). Sex-differences in timing and context of dispersal in Damaraland mole-rats (Fukomys damarensis). J. Zool. 306, 252-257.

Torrents-Ticó, M., Bennett, N.C., Jarvis, J.U.M. \& Zottl, M. (2018b). Growth affects dispersal success in social mole-rats, but not the duration of philopatry. Biol. Lett. 14, 20180005.

Young, A.J. \& Bennett, N.C. (2010). Morphological divergence of breeders and helpers in wild damaraland mole-rat societies. Evolution (N. Y). 64, 3190-3197.

Young, A.J., Jarvis, J.U.M., Barnaville, J. \& Bennett, N.C. (2015). Workforce effects and the evolution of complex sociality in wild Damaraland mole rats. Am. Nat. 186.

Zöttl, M., Thorley, J., Gaynor, D., Bennett, N.C. \& Clutton-Brock, T. (2016a). Variation in growth of Damaraland mole-rats is explained by competition rather than by functional specialization for different tasks. Biol. Lett. 12, 20160820.

Zöttl, M., Vullioud, P., Mendonça, R., Torrents Ticó, M., Gaynor, D., Mitchell, A. \& Clutton-Brock, T. (2016b). Differences in cooperative behavior among Damaraland mole rats are consequences of an age-related polyethism. Proc. Natl. Acad. Sci. 113, 201607885.

Zöttl, M., Vullioud, P., Goddard, K., Torrents-Ticó, M., Gaynor, D., Bennett, N.C. \& Clutton-brock, T. (2018). Allo-parental care in Damaraland mole-rats is female biased and age dependent, though independent of testosterone levels. Physiol. Behav. 193, 149-153. 


\section{ETHICS}

621 The research carried out in this study was approved by the University of Pretoria Animal Ethics

622 Committee (ECO32-13, EC050-16)

\section{ACKNOWLEDGEMENTS}

624 We are grateful to numerous field assistants that helped to collect the field data (usually under

625 hot and/or nocturnal conditions), and to numerous research managers and onsite employees 626 who have contributed to the long-term running of the Kalahari mole-rat project. In particular, 627 we would like to acknowledge Philippe Vullioud, David Gaynor, Tim Vink, Walter Jubber, 628 and Nigel Bennett for various logistical and academic input to the project. We are also indebted 629 to the Kalahari Research Trust for access to the facilities, and to Prof. Marta Manser for her 630 contributions and efforts towards running and maintaining the Kalahari Research Centre, 631 supported by funding from the University of Zurich and the MAVA foundation. Lastly, we 632 thank the Northern Cape Department of Environment and Nature Conservation for permission 633 to work in the Northern Cape, and Kobus Lamprechts for allowing us to work on his land. This 634 work was supported by grants from Vetenskapsrådet (2017-05296) and Crafoordska Stiftelsen 635 (2018-2259 \& 2020-0976) awarded to M.Z., and grants from the European Research Council 636 (European Union's Horizon 2020 research and innovation program, no. 742808 and no. 637 294494) awarded to T.C-B.

\section{AUTHOR CONTRIBUTIONS}

639 J.T, H.B, and M.Z planned and designed the study. J.T. carried out statistical analyses with 640 input from H.B and M.Z. J.T., H.B, M.Z., and K.F conducted fieldwork and maintained the 641 database. T.C-B. provided access to facilities and infrastructure at the Kuruman River Reserve. 642 J.T. and M.Z wrote the manuscript, with input from all co-authors throughout. 

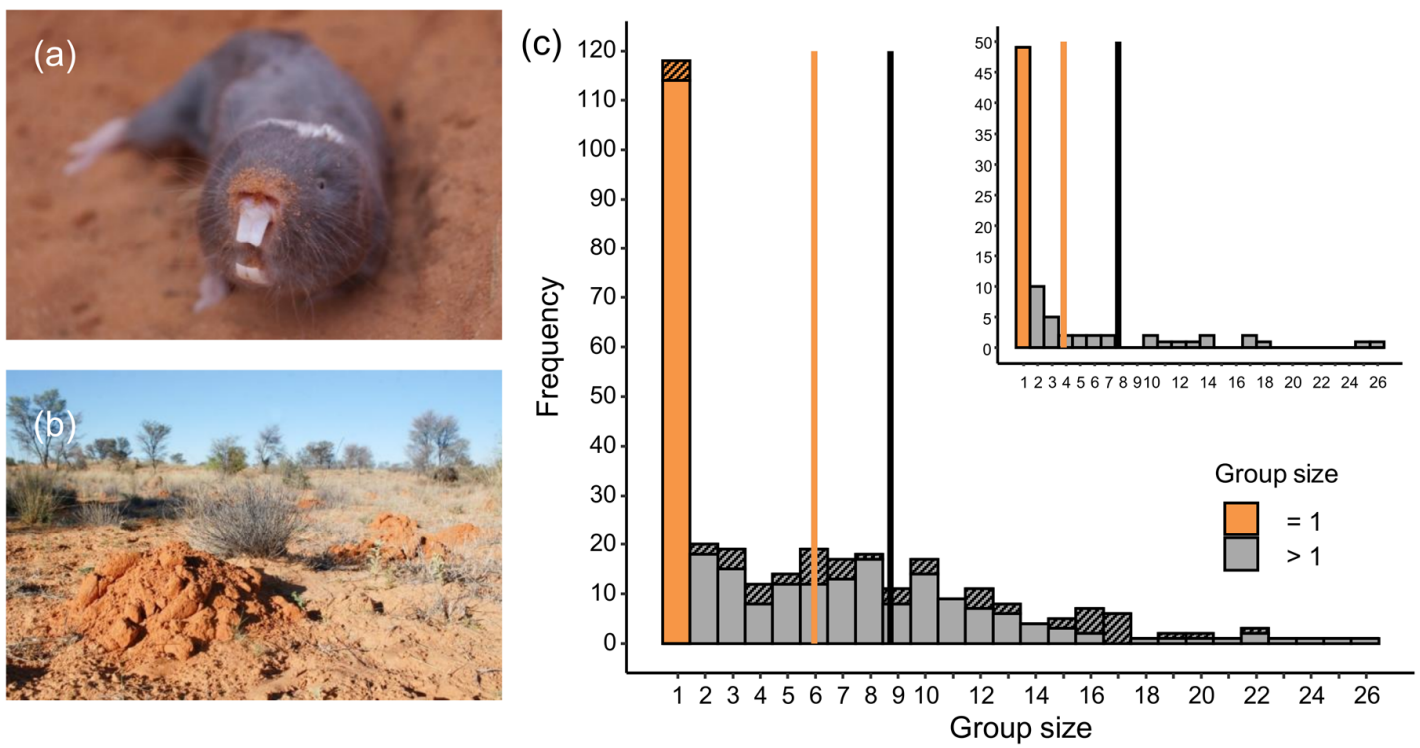

644 Figure 1. Group sizes for Damaraland mole-rats in the southern Kalahari. (a) An adult

645 Damaraland mole-rat. (b) A line of sand mounds that is created by a group of mole-rats when

646 they dig foraging tunnels underground. (c) The distribution of group sizes in the study

647 population at the Kuruman River Reserve. The main histogram displays group size frequencies

648 across all captures, while the inset histogram displays the group size at first capture for each

649 unique group ( $\mathrm{n}=82$; including single individuals). In each case, a large proportion of the

650 population were captured as single individuals (orange), who were almost exclusively females.

651 The vertical lines display the mean group size either including (orange) or excluding (black)

652 these single individuals, and for all captures, we distinguish between complete and incomplete

653 group captures by hatching the latter (mean for all complete group captures $=5.45,1 \mathrm{SD}=5.51$;

654 mean for complete captures of groups $>1=8.54,1 \mathrm{SD}=5.32$ ). Removing the $15.2 \%$ of

655 incomplete captures had limited influence on the mean group size (not displayed). 


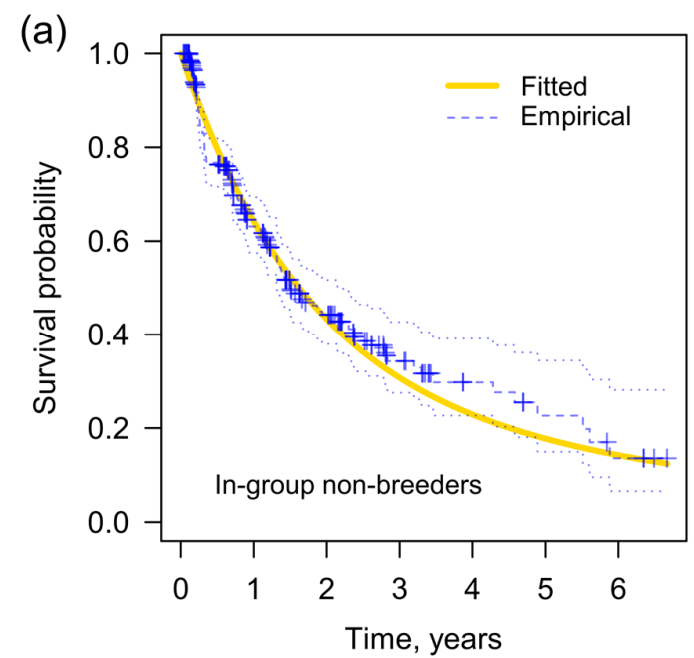

(b)
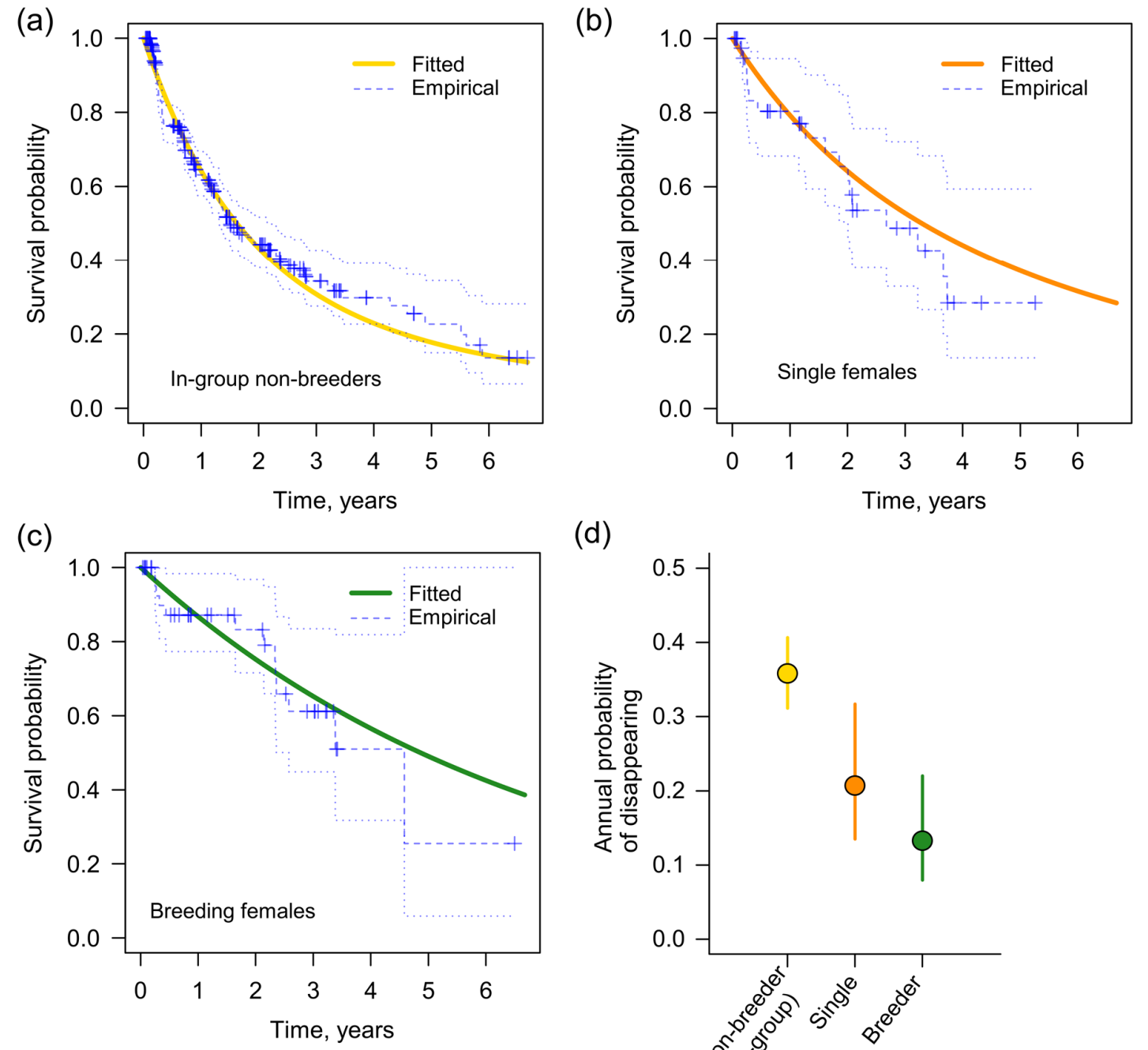

(d)

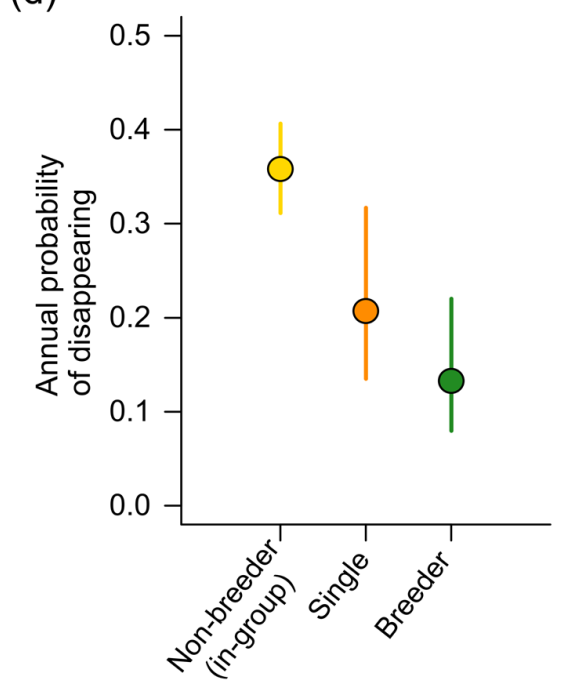

Figure 2. Status-related survivorship in female Damaraland mole-rats: in-group non-

658 breeders (a), single females (b), and breeding females (c). Solid lines display the expected 659 probability of survival from each state and dotted lines display the 'empirical' Kaplan-Meier 660 estimate of survival probability, with crosses denoting cases of censorship. Here, survival strictly refers to disappearance from the study population, and the predicted annual probability of disappearance (mean $\pm 95 \% \mathrm{CI}$ ) is given in (d). 'Survival' therefore combines cases of instate mortality with cases of dispersal, where individuals have left their current group and state and are not re-captured thereafter. Dispersal is most likely to account for a large proportion of disappearances from the in-group non-breeder state, and the low incidence of recaptures of individuals thereafter implies that dispersal carries a high cost of mortality. Note that one

667 female had been recaptured as a singleton for 5.26 years by the end-point of the study, and was right-censored at that point. 
(a)

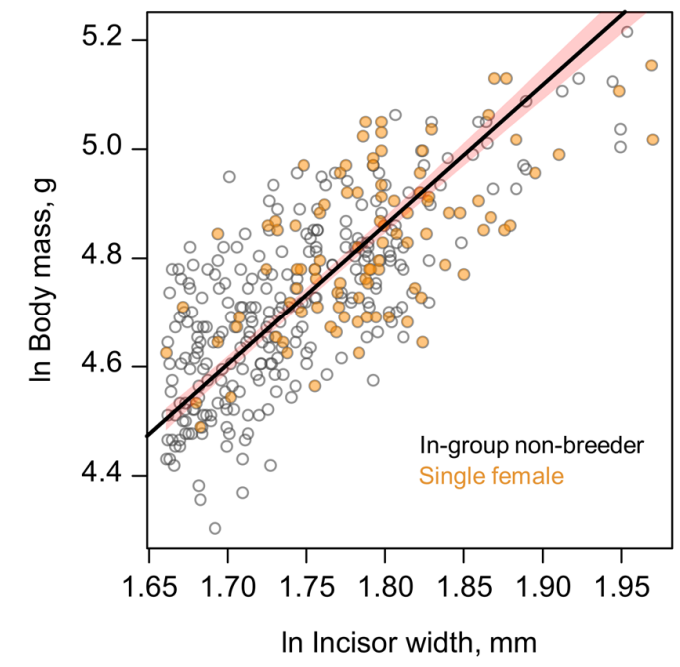

(b)

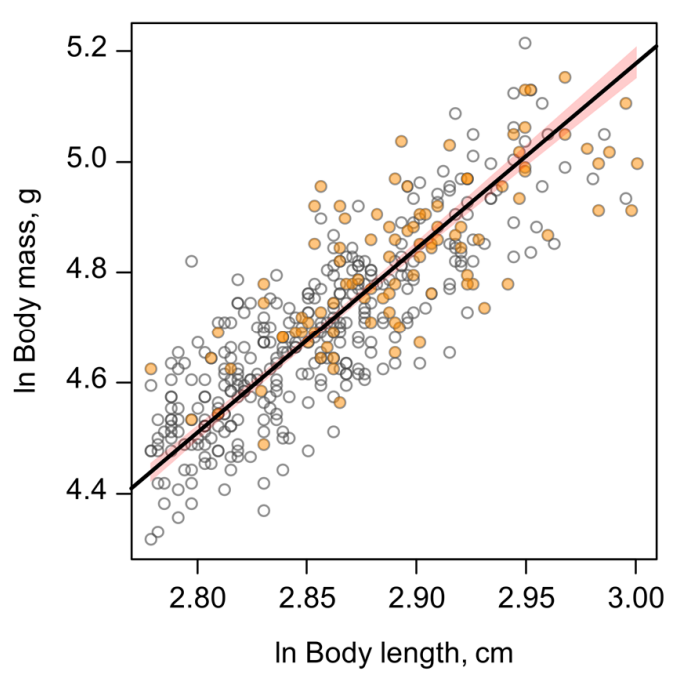

669

670

671

672

673

674

675

676

677

Figure 3. The body condition of dispersed single females compared to size-matched ingroup non-breeding females. The principal plots display the scaled major axis regression (SMA) between body mass and incisor width (a), and between body mass and body length (b). Points display the raw data and lines display the predicted regression through all the data, with 95\% bootstrapped confidence intervals given by the shaded area. Allowing the two classes of female to have different intercepts and slopes did not improve model fit: the two classes of 676 female did not differ in mean body condition, or in the change in body condition with increasing 77 skeletal size. 
(a)

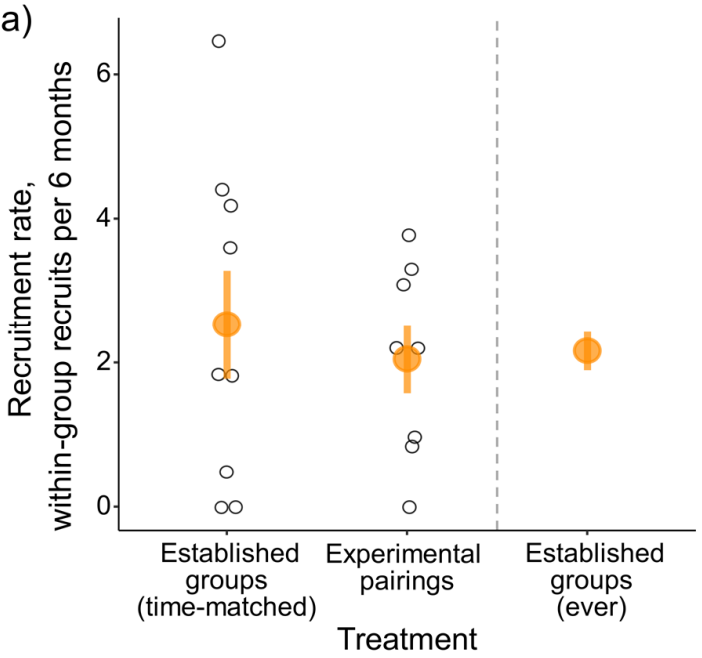

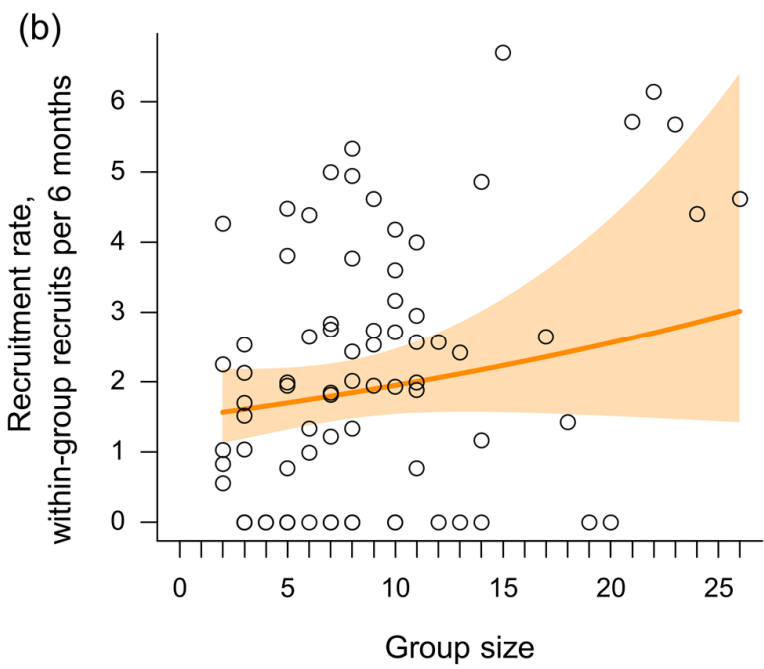

678

679 Figure 4. The effect of group size on within-group recruitment. a) The recruitment rate of established groups did not differ from that of newly created pairs when measured over the same

681 time period. The mean rate of recruitment in this experimental period (middle panel in 5a) was 682 similar to the overall mean recruitment rate in the population (right panel in 5a; average of all 683 values in $5 \mathrm{~b}$ ). The larger orange points denote the treatment mean \pm 1 SEM. b) In contrast, 684 longitudinal analyses of group size across the duration of study detected a modest effect of 685 increasing group size on the rate of within-group recruitment $(0.216 \pm 0.106, p=0.042$, 686 GLMM, link-scale); solid line shows the predicted mean and shading shows the 95\% 687 confidence intervals. In both panels the black points give raw data, corrected for the trapping 688 interval duration, and in all cases, recruitment has been standardised to a 6-monthly rate 689 according to the time difference between the first capture and the second capture. 
(a)

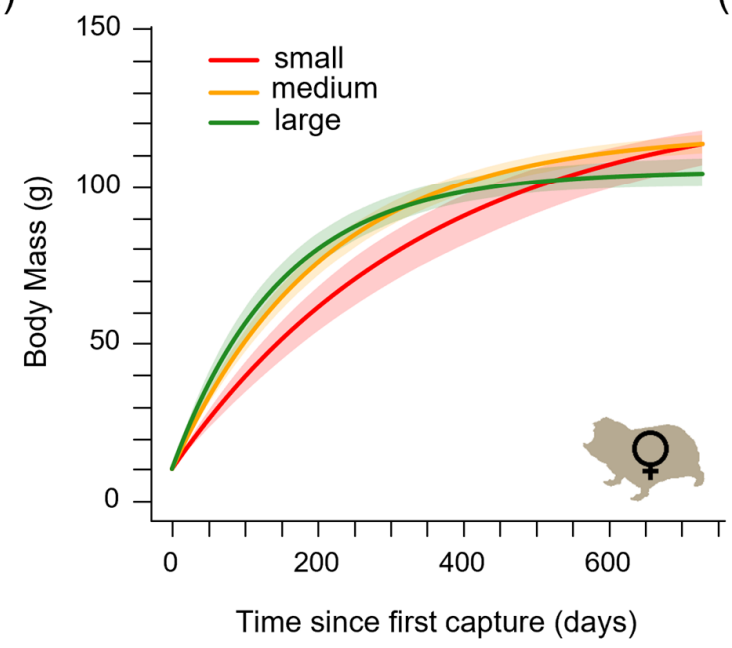

(b)

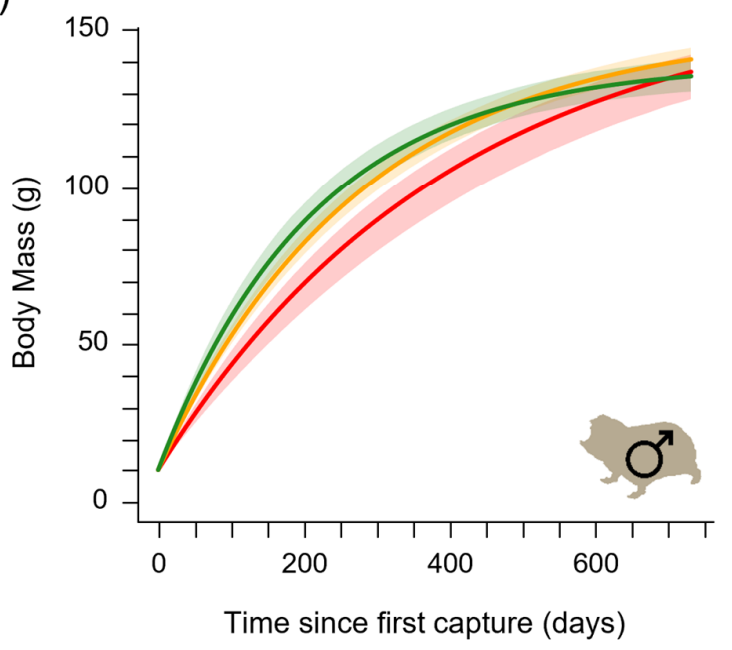

690 Figure 5. The effect on group size on growth of wild Damaraland mole-rats. Body mass

691 growth depends upon whether the individual was growing in a small (4 individuals, red),

692 medium (12 individuals, orange), or large (20 individuals, green) group. (a) and (b) display the 693 predicted body mass growth of a male or female mole-rat first captured at 10g, the average size 694 of a pup at parturition. Curves are derived from von Bertanlanffy interval equations and show 695 the predicted mean mass, with the shaded areas indicating 95\% confidence intervals. 


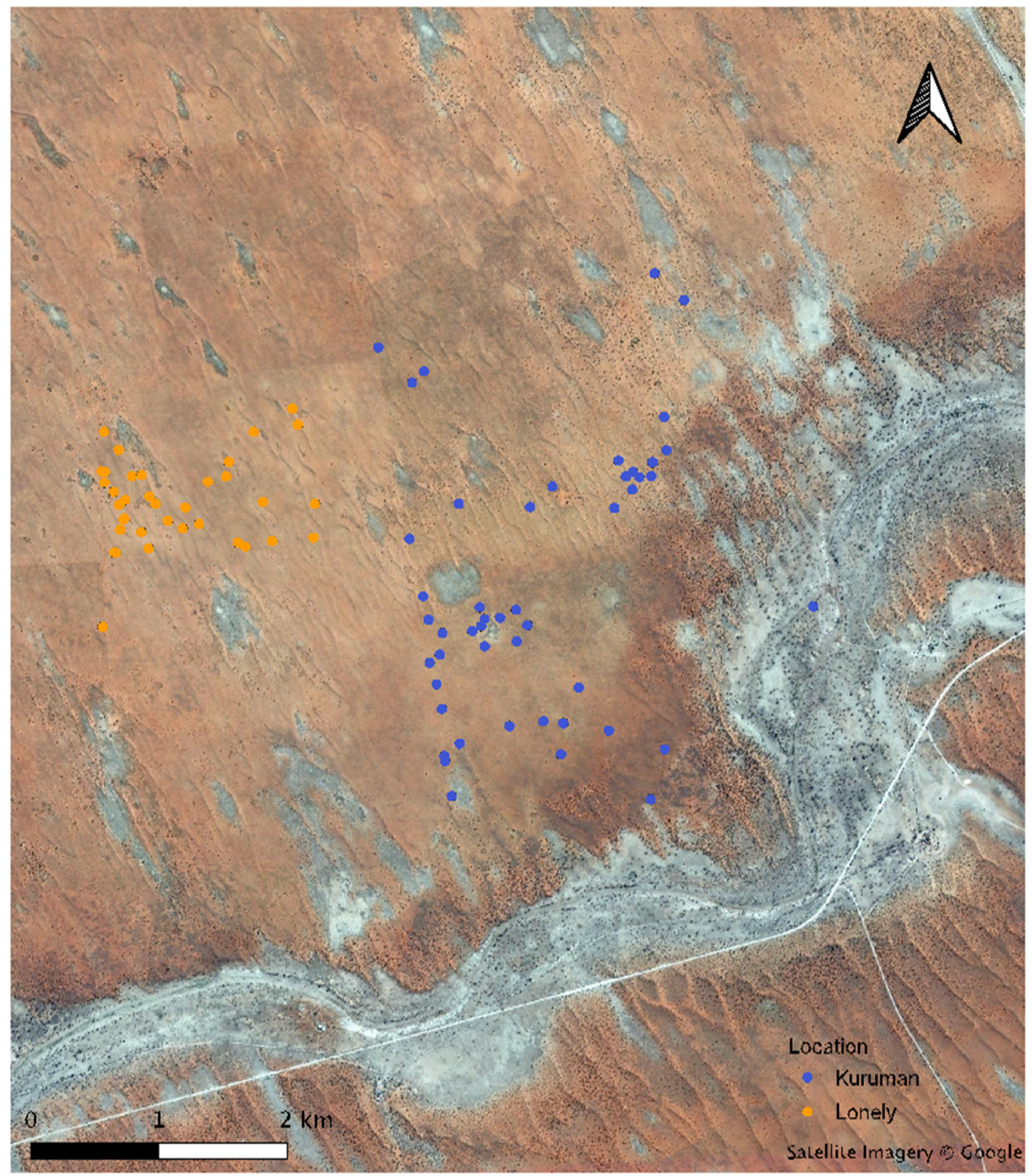

Figure S1. The trapping locations of Damaraland mole-rats groups captured at the Kuruman River Reserve and Lonely farm between 2013 and 2020. Each group is noted by a point which marks the centroid of all group captures, with the point colour corresponding to location, "Kuruman" or "Lonely". Note that with only one exception, mole-rat groups were distributed entirely within the red 'arenosol' soils, where their principal food (Gemsbok cucumber) is found. The one exception to this pattern was a single dispersing female who temporarily burrowed in an area of calcareous soils, which appear light grey from satellite. These calcareous soils highlight the path of the long dried up Kuruman river as it passes through the reserve. 
bioRxiv preprint doi: https://doi.org/10.1101/2021.12.08.471794; this version posted December 10,2021 . The copyright holder for this preprint (which was not certified by peer review) is the author/funder, who has granted bioRxiv a license to display the preprint in perpetuity. It is made available under aCC-BY-NC-ND 4.0 International license.

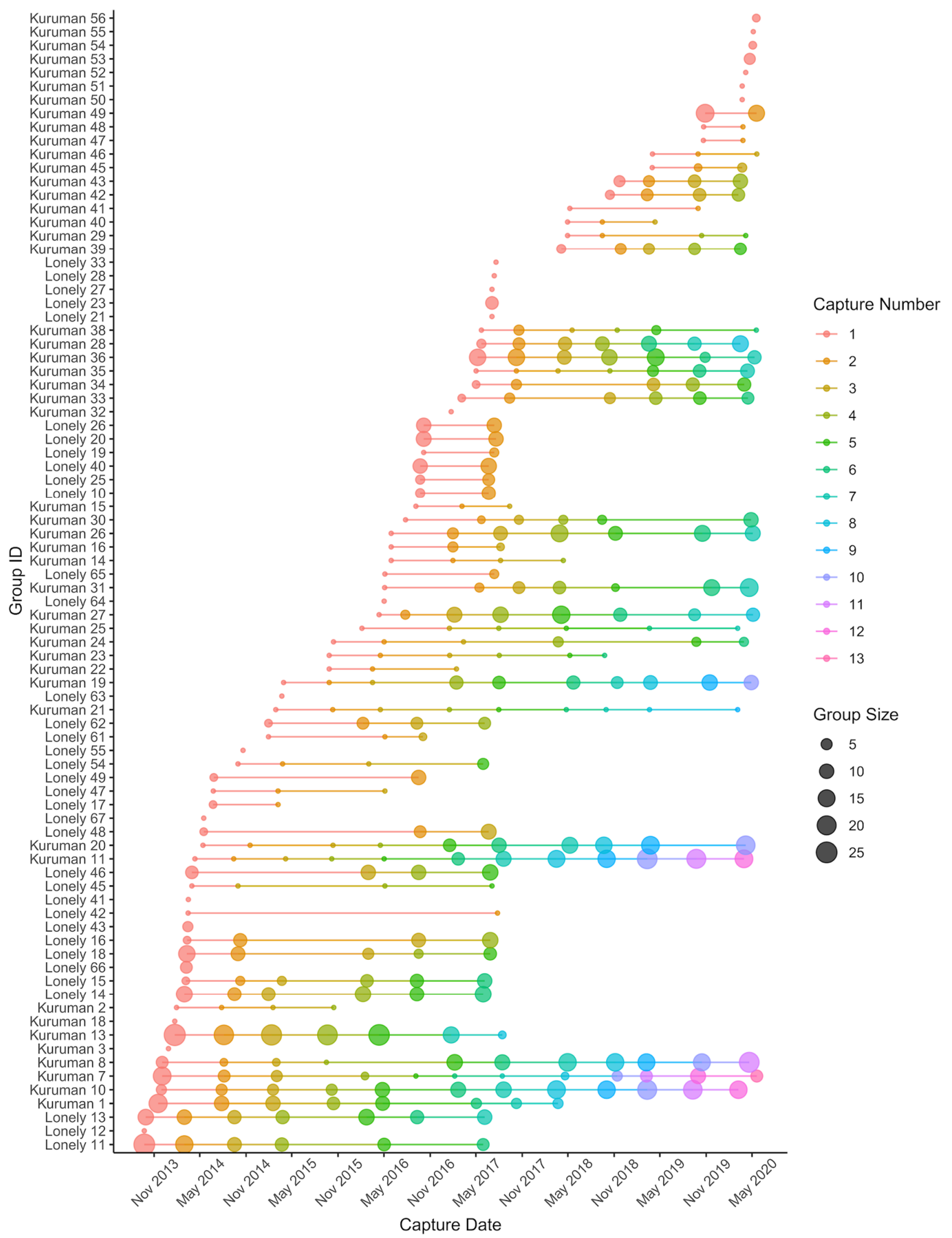

Figure S2. The history of trapping effort of Damaraland mole-rats groups in and around

the Kuruman River Reserve. Each unique group has its own row, where successive capture events are denoted by points moving right along the $\mathrm{x}$-axis. The size of the points is proportional to the size of the group at each capture event. 


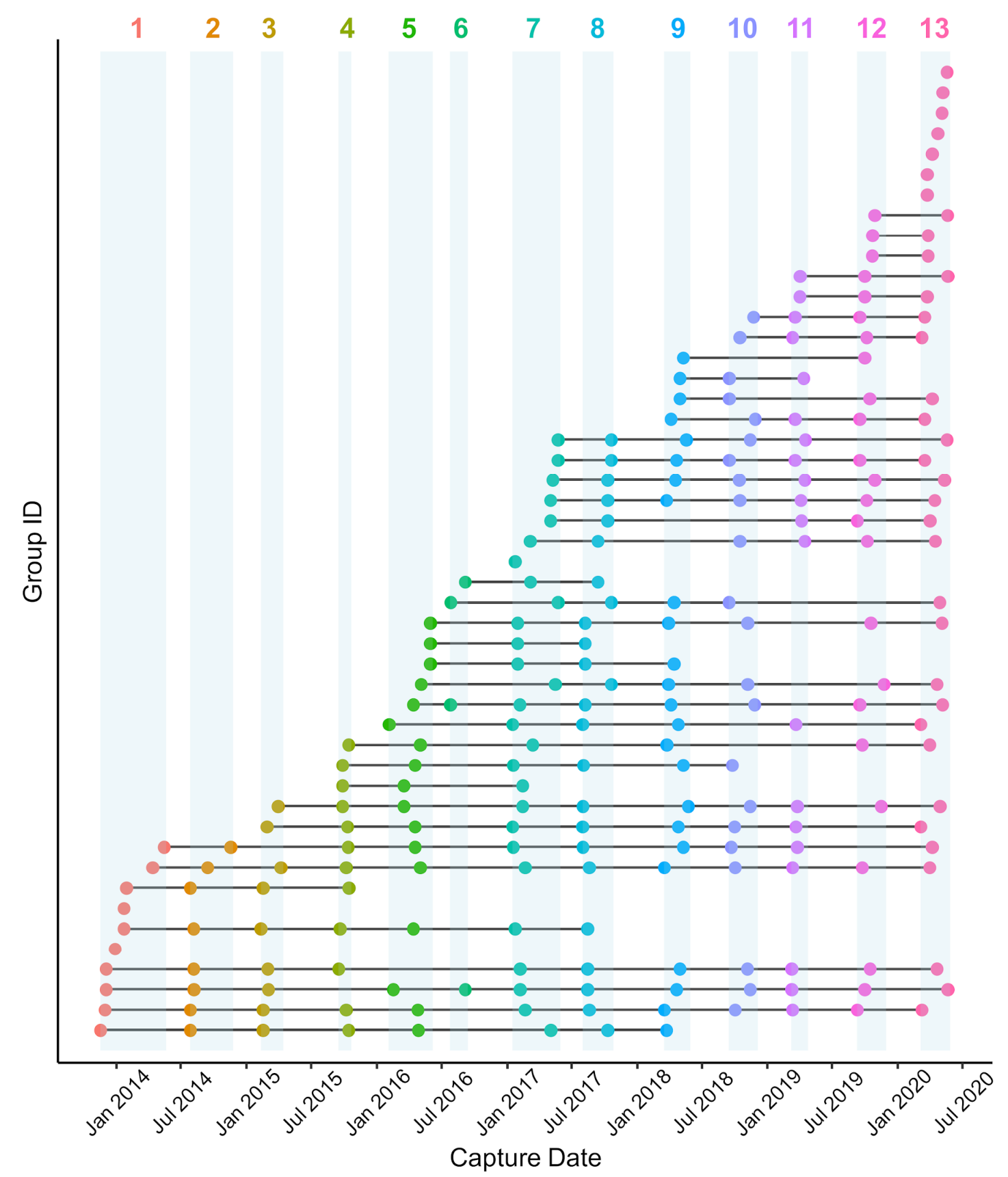

Figure S3. The history of trapping effort for Damaraland mole-rat groups at the Kuruman River Reserve in the Kalahari, separated into discrete trapping windows. Periods are defined by any break in trapping effort of more than 2 months. By doing so, it can be seen that no group is captured twice within a trapping period. Also, most groups are captured in successive trapping periods, with very few groups 'skipping' trapping windows- with the exception of period 6, where only a few groups were recaptured. In the few other cases where groups skips a trapping period, this is because no mounds were visible throughout the period, and when this occurs, it is not known whether a group had collapsed/disappeared or was present underground but not digging. 


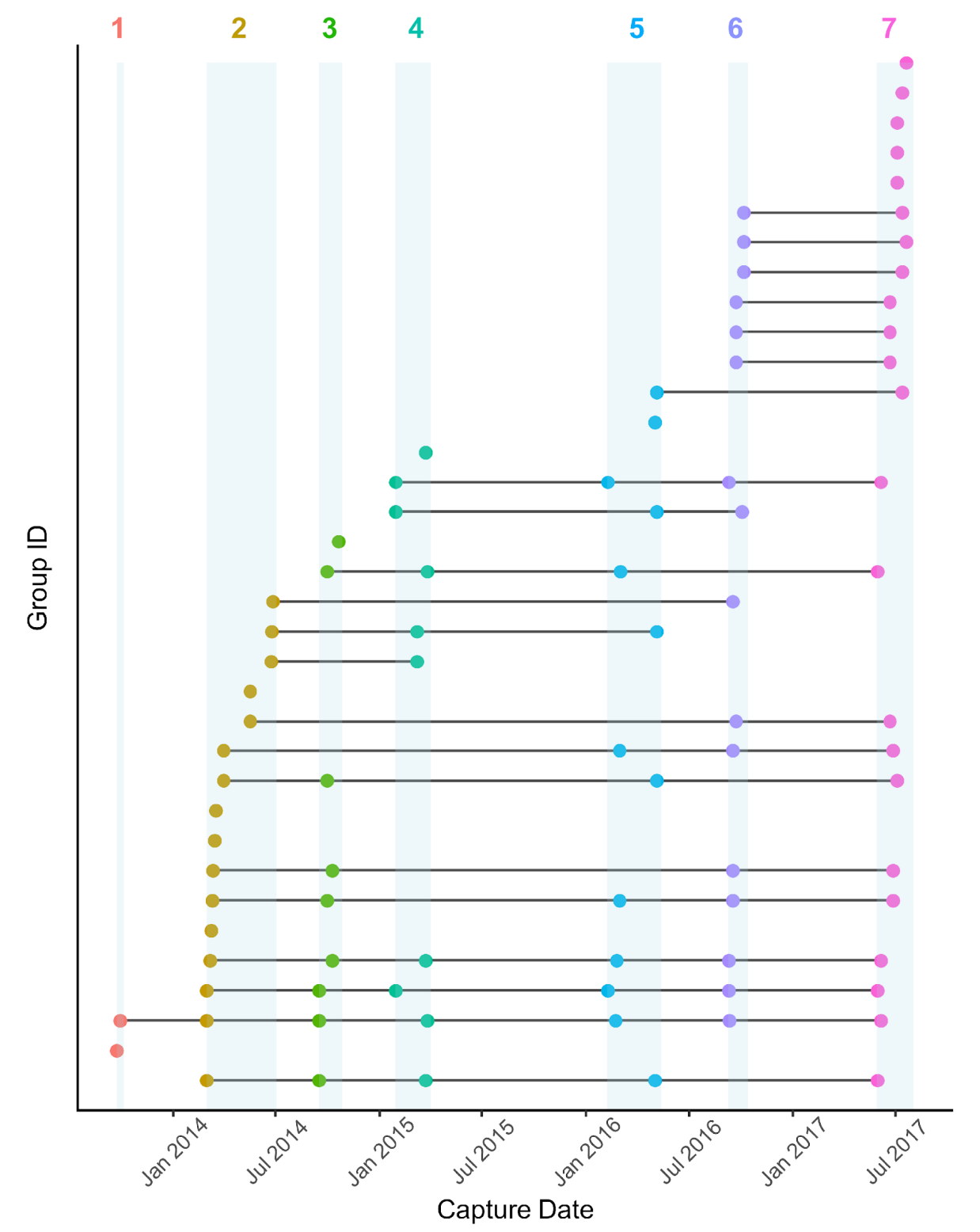
the Kalahari, separated into discrete trapping windows. Periods are defined by any break in trapping effort of more than 2 months. By doing so, it can be seen that no group is captured twice within a trapping period. Also, most groups are captured in successive trapping periods, with very few groups 'skipping' trapping windows- with the exception of period 6, where only a few groups were recaptured. In the few other cases where groups skips a trapping period, this is because no mounds were visible throughout the period, and when this occurs, it is not known whether a group had collapsed/disappeared or was present underground but not digging. 


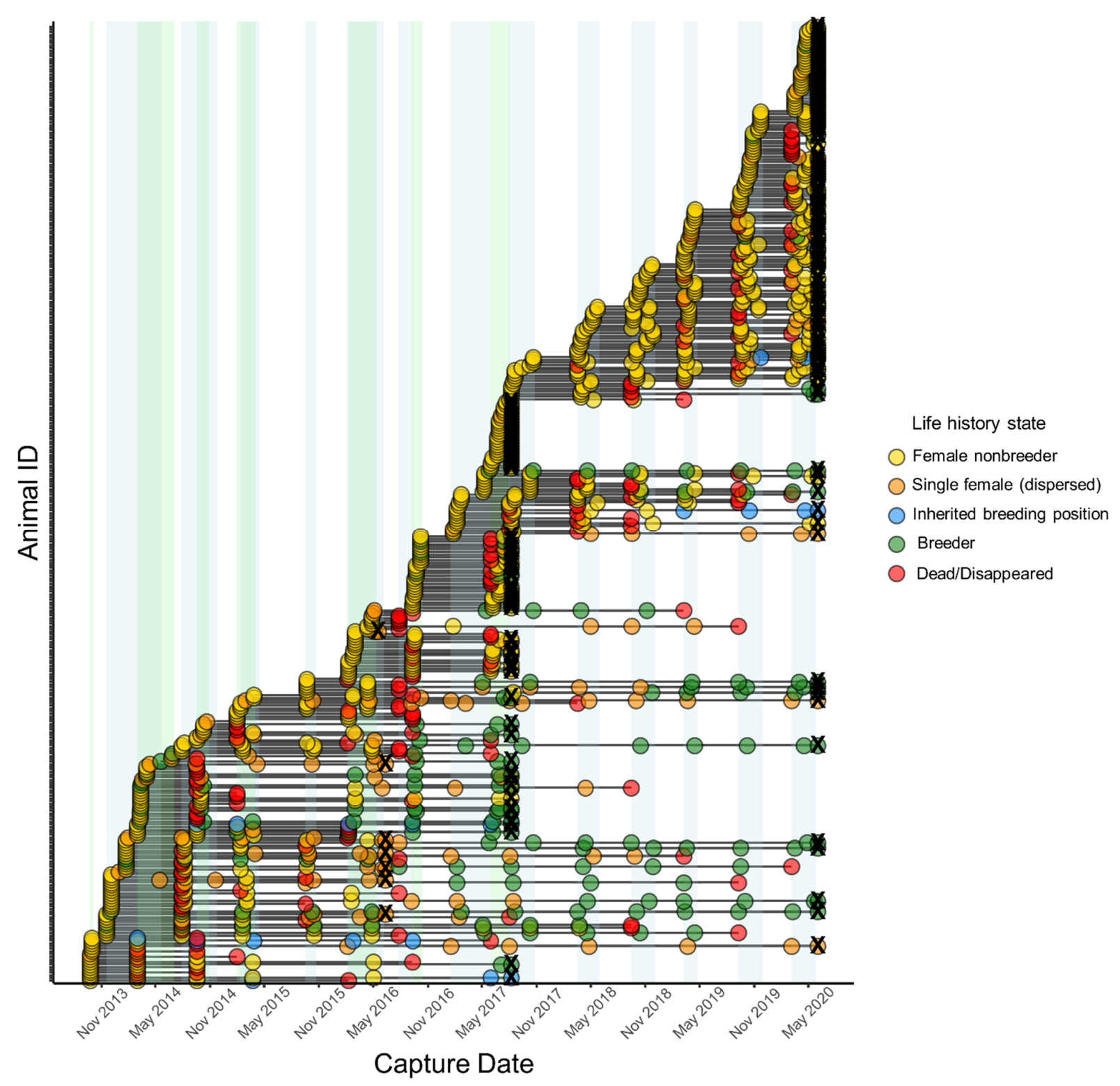

Figure S5. The fates of female Damaraland mole-rats capture from 2013-2020. Though analysed together, the fate/current state of female non-breeders at the Kuruman River Reserve and Lonely were assessed separately since they took advantage of different trapping windows (KRR- blue shaded areas; Lonely; green shaded areas). Individual states at any given time are female non-breeder, single female (who has dispersed from her natal group), an out-of-natal group breeder (again dispersed), and disappeared/died. In a small number of cases females also acquired dominance in their natal group (when an immigrant male had moved in previously). For individuals that were not recaptured at some point before the final trapping period in either location, it was assumed that the individual had died or disappeared at some point between their last capture and start of the next trapping window; as handled by the models. Additionally, individuals captured in the last trapping period at each location were assumed to have persisted 746 in their current state to the end of the period, where they were right-censored $(\mathbf{X})$. 
747

748

749

750

751

752

753

754

755

756

757

758

759

760

761

762

763

764

765

766

767

768

769

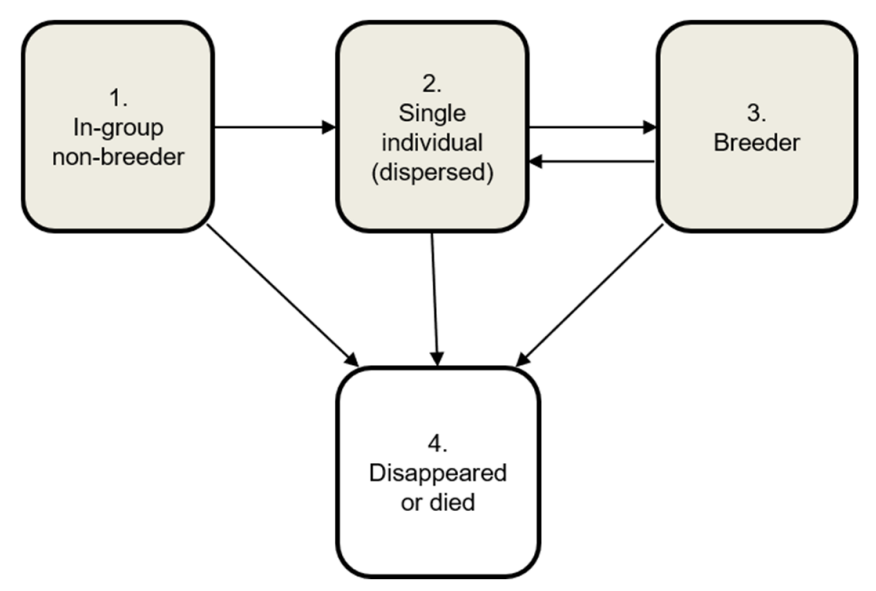

$$
Q=\left(\begin{array}{cccc}
q_{11} & q_{12} & 0 & q_{14} \\
0 & q_{22} & q_{23} & q_{24} \\
0 & 0 & q_{33} & q_{34} \\
0 & 0 & 0 & 0
\end{array}\right)
$$

Figure S6. Multi-state model for the life history transitions of female Damaraland molerats, with associated $\mathbf{Q}$ matrix. In a small number of cases where individuals were experimentally paired (i.e. given a male when formerly present as a single female and analysed in other papers; e.g. Thorley et al. 2018 Proc. R. Soc. B, 285: 20180897), they were also rightcensored at the point of pairing so that any transitions from single female to breeder did not include this experimental component. In all cases, the multi-state model was parameterised so that the timing between transitions was not known exactly, but was estimated under Markov assumptions- this includes the timing of death/disappearance. 
770

771

772

773

774

775

776

777

778

779

780

781

782

783

784

785

786

787

788

789

790

791

792

793

794

795

796

797

798

799

800

801

802

803

804

805

806

807

808

809

810

811

812

813

814

815

816

\section{The condition of single females}

To compare the body condition of single females and in-group non-breeding females we performed standardised major axis regressions of body mass on two skeletal size traits: incisor width and body length. We included body length as this is a more conventional linear measurement of skeletal size in mammals. Incisor width was measured as noted in the main text. Body length was measured dorsally from the front of the snout to the tip of the tail using a tape measure (to an accuracy of $1 \mathrm{~mm}$ ). As for incisor width, each measurement was taken in duplicate by two observers, and we here use the average of these two measures. For each skeletal measurement we filtered out any in-group non-breeding females whose skeletal size was less than the minimum size trait found in single females. This meant that the two classes of female were size-matched skeletally, such that any difference in condition would reflect increased bodily reserves for a given skeletal size.

Standardised major axis regression was proposed by Peig \& Green (2008) as a superior regression method for the scaling of mass on linear body measurements than ordinary least squares methods, and has been widely adopted in studies on mammals since. Briefly, we performed a standardised major axis regression of ln body mass against the natural log of each skeletal measurement using the smatr package (Warton et al. 2012). In both cases, we initially fitted a model which allowed the slope and intercept to vary according the class of female, but for both skeletal measurement neither the intercept (teeth width LRT: $\chi^{2}{ }_{1}=1.04, p=0.31$; body length LRT: $\chi^{2}{ }_{1}=0.09, \mathrm{p}=0.76$ ) nor the slope (teeth width LRT: $\chi^{2}{ }_{1}=1.31, \mathrm{p}=0.25$; body length width LRT: $\chi^{2}{ }_{1}=2.53, \mathrm{p}=0.11$ ) significantly different between single females and in-group non-breeders. This indicates that single females and in-group non-breeders did not differ in their condition.

We therefore present the results of the scaling relationship where all females were assumed to following the same scaling relationship; modelled by a single slope and intercept (Figure 3 in main manuscript, incisor width intercept $=2.41,95 \% \mathrm{CI}=-0.08-0.56$, incisor width slope $=2.57,95 \% \mathrm{CI}=2.39-2.75$; body length intercept $=-4.83,95 \% \mathrm{CI}=-5.33-$ 4.33 , body length slope $=3.34,95 \% \mathrm{CI}=3.17-3.52$ ).

The scaled mass index of each measurement can be calculated using the following formula:

$$
S M I=M_{i}\left(\frac{L_{0}}{L_{i}}\right)^{b_{S M A}}
$$

Where $M_{\mathrm{i}}$ and $L_{\mathrm{i}}$ are the body mass and skeletal measurement of individual $i, b_{S M A}$ is the scaling exponent estimated by the SMA regression of $M$ on $L$, and $L_{0}$ is the arithmetic mean value of the population sample. The scaled mass index of single females and in-group non-breeding females are contrasted below in the inset boxplots.

\section{References:}

Peig, J. \& Green, A.J. (2009) New perspectives for estimating body condition from mass/length data: the scaled mass index as an alternative method. Oikos 118, 1883-1891.

Warton, D.I., Duursma, R.A., Falster, D.S., \& Taskinen, S. (2012) smatr 3- an R package for estimation of allometric lines. Methods in Ecology and Evolution 3(2), 257-259. 


\section{Experimental Pairings}

818
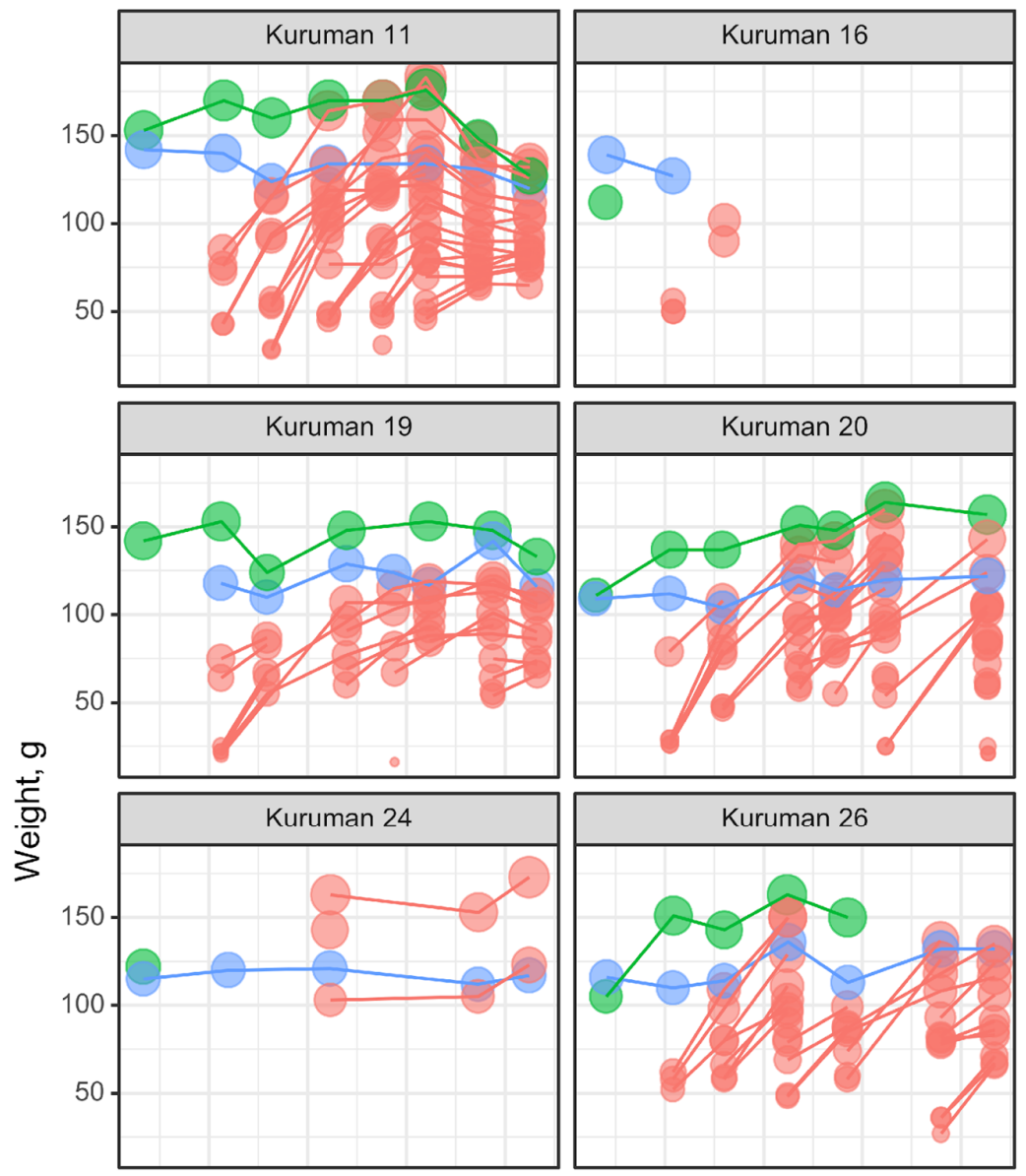

weight

○ 50

100

150

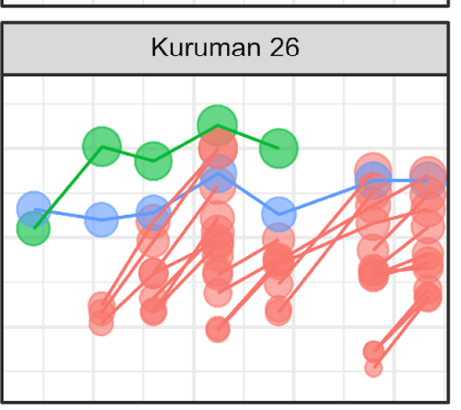

breeder

- Other

- Paired King

$\rightarrow$ Initial Solitary Female
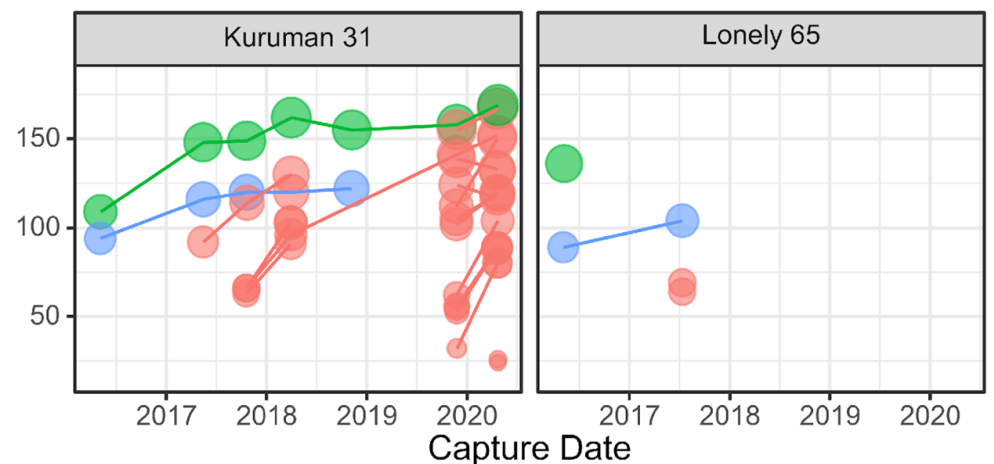

Figure S7. Group structure and trapping history of groups that were created experimentally with the addition of an unfamiliar male to the burrow systems of single 

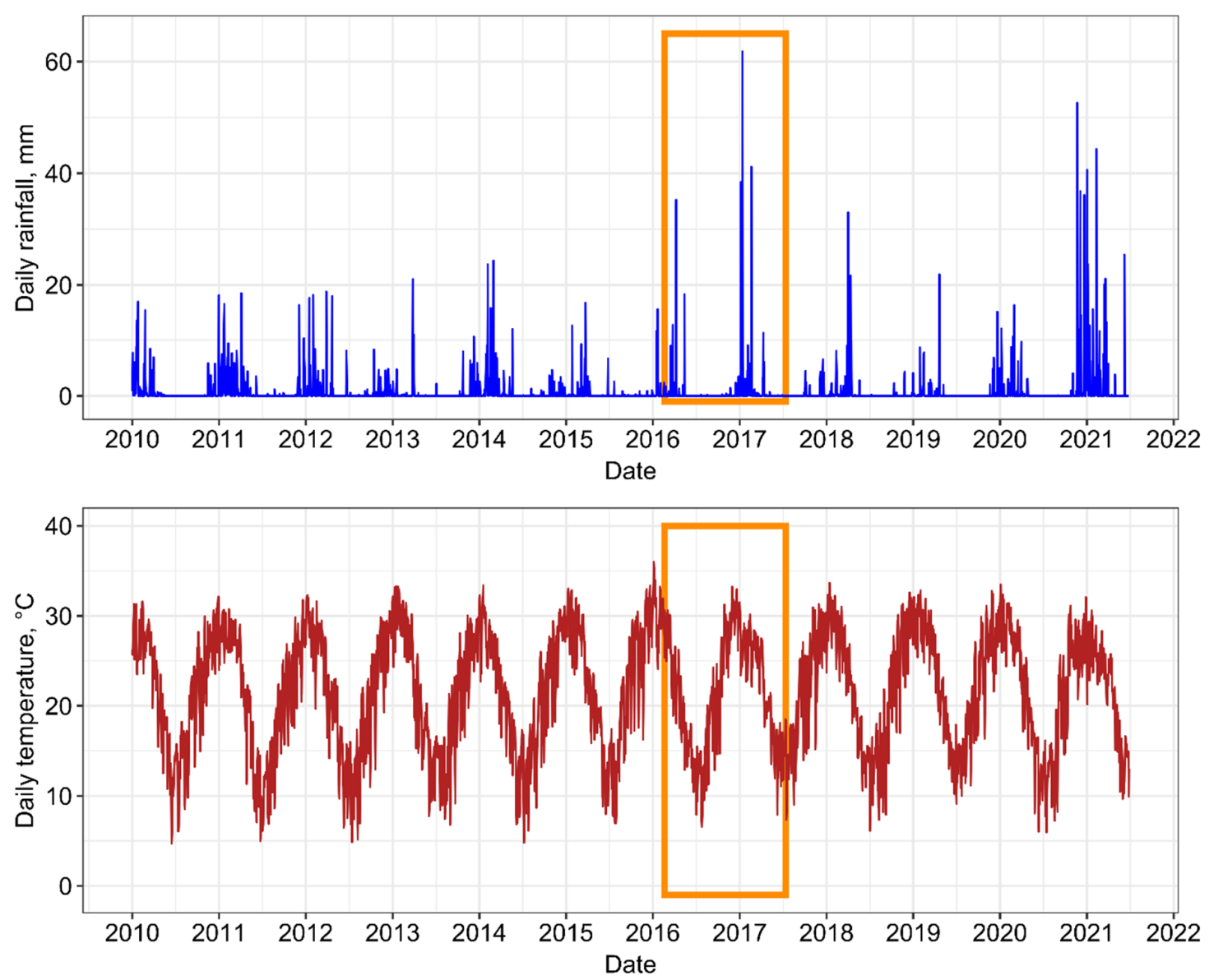

824
Figure S8. The timing of the experimental pairings in relation to climatic variation at the Kuruman River Reserve. Upper and lower panels document the long-term daily rainfall ( $\mathrm{mm}$ ) and daily average temperature at the field site. The orange rectangle demarcates the period within which experimentally created pairs and established groups (which served as controls) were captured and recaptured. Climate data taken from NASA's GMAO MERRA-2 assimilation model and GEOS 5.12.4 FP-IT. 
(a)

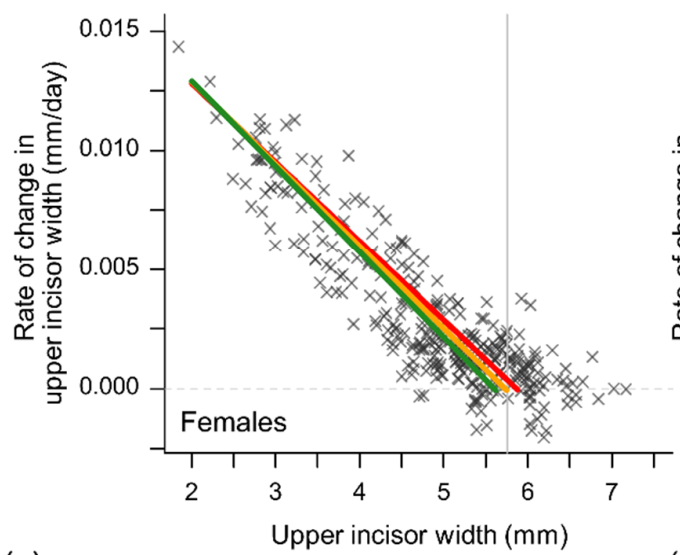

(c)

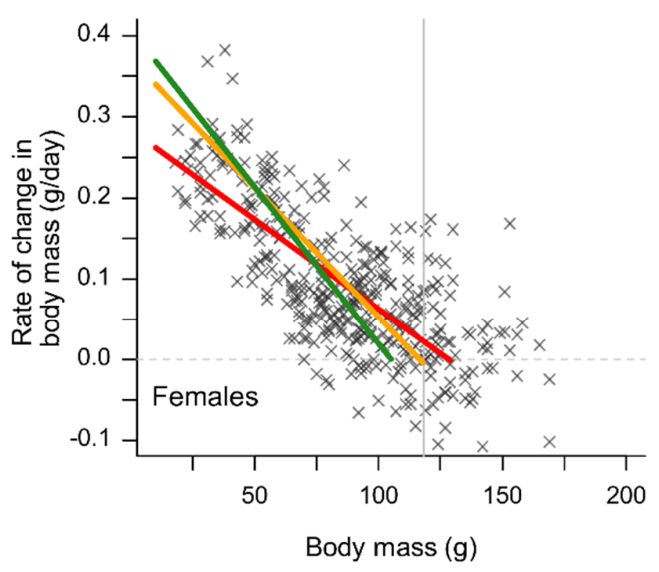

(b)

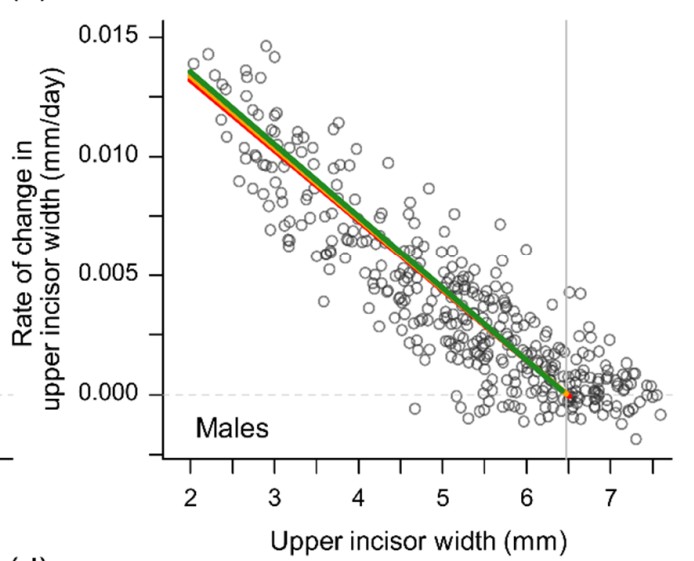

(d)

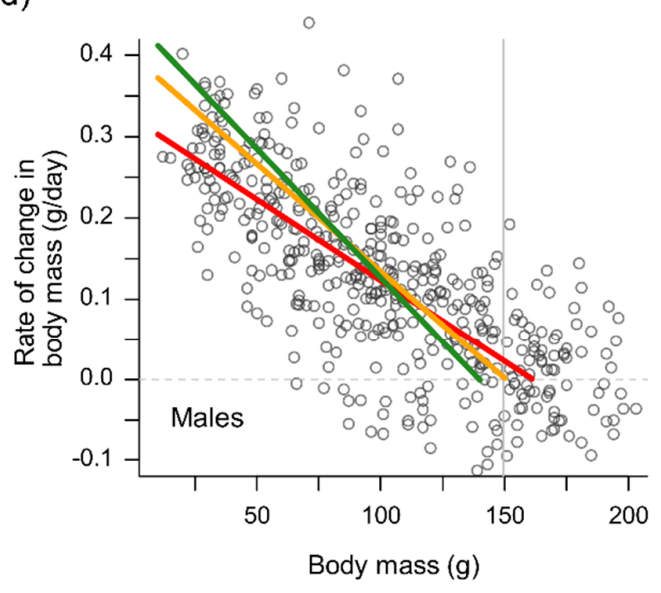

Figure S9.The rate of change in incisor width and body mass for females (a, c) and males (b,d) with variation in initial size and group size. Each point represents the change in incisor width or body mass across two captures - a capture and a recapture. Note that with increasing initial size, the slope of the change in size metric converges on the estimated population-level asymptotes (vertical lines). 


\section{Group size and adult body mass}

839

840

841

842

843

844

845

846

847

848

849

850

851

852

853

854

855

856

857

858

859

860

861

862

863

864

865

866

867

Because the parameters of the von-Bertalanffy growth curve covary with one another (as parameterised), it is not possible to dissociate the effect of group size on the growth rate constant, $k$, from the effect of group size on asymptotic mass, $A ; k$ itself contributes to the estimation of $A$. We therefore carried out additional analyses to clarify the effect of group size on adult body mass.

For all the males and females whose growth was modelled by the interval equations (Figure S7), we extracted weight information for all the times that they were captured at least one year after first being captured at less than 1 year of age $(<100 \mathrm{~g}$ for males, $<80 \mathrm{~g}$ for females). The weights information therefore represented the body mass of all non-breeding individuals captured beyond 1 year of age, with most data coming from individuals far older than this. This generated a data set of 152 weights from 73 males, and 111 weights from 65 females. At the same time, we calculated the average group size that each of these individuals had experienced in their first year life; for some individuals we had only one value across this period, for others we had up to 3 measures.

The mass of individuals in adulthood of either sex was then modelled according to their current group size and the group size that they experienced in early life ( $<1$ year of age). The two terms were not strongly correlated ( $r=0.26$ in males, $r=0.04$ in females), and in both the male and the female data sets the variance inflation factor of the two terms was $<1.07$. Both early life group size and adult group size could therefore be fitted together in the same model. Adult body mass was fitted in a linear mixed effects model (Gaussian error), with average group size in the first year specified as a continuous variable, and group size in adulthood specified as a categorical variable with three levels: small, medium, and large, separated into the tertiles in each data set. For males at 1-9 (small), 10-16 (medium), >16 (large); for females at 1-7 (small), 8-15 (medium), >15 (large). An additional variable noting the quarterly period of the year was also included (Jan-Mar; Apr-Jun; Jul-Sep; Oct-De). Group size in early life was standardised prior to model fitting, and a random effect of individual identity, nested within group identity, was specified in each case. Models were fitted in the lme4 package in R. Model outputs are presented in tables S9, and visualised in Figure S8. 
(a)
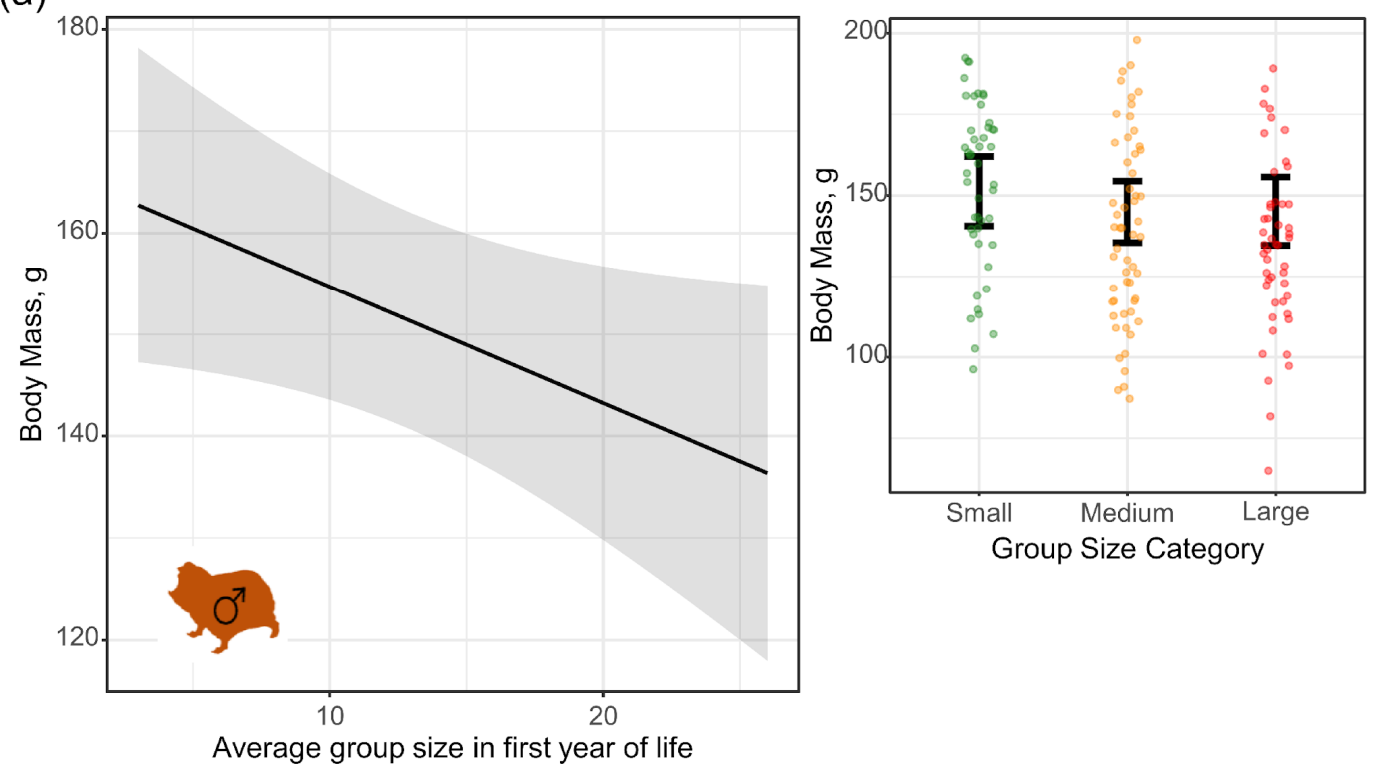

(b)
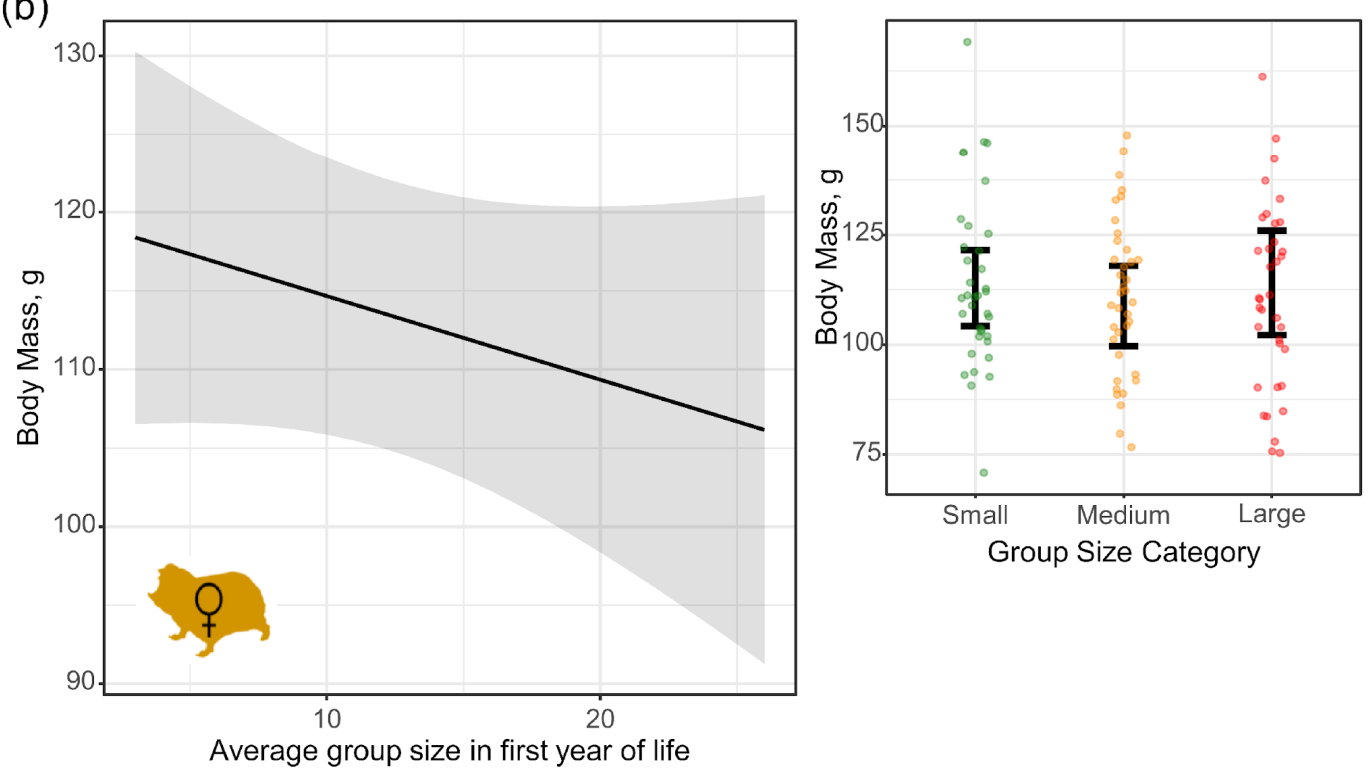

Figure S10. Adult body mass of non-breeding male (a) and female (b) Damaraland molerats. Left panels display the predicted effect of average group size in the first year of life on adult mass ( $<100 \mathrm{~g}$ for males, $<80 \mathrm{~g}$ for females), as estimated from linear mixed effects models (table S11). In both cases, increases in group size during early life reduces body mass in adulthood. By contrast, current group size in adulthood has no effect on adult body mass, as displayed in the right panels. Points denote the raw data across all individuals, with error bars denoting the predicted marginal effects across three group size categories. Group size categories were separated at the tertiles within each data set; for males at 1-9 (small), 10-16 (medium), >16 (large); for females at 1-7 (small), 8-15 (medium), >15 (large). Post-hoc tests performed on the linear mixed effects models found that in neither sex did group size in adulthood have a significant effect on adult body mass ( $p>0.46$ in all contrasts). Note that group size in adulthood was categorised largely for the purpose of visualisation; treating the term as a continuous variable did not qualitatively affect the results. 
Table S1. Sum of captures at the Kuruman River Reserve for each trapping period. Recapture rate was calculated as the number of recaptured individuals divided by the total number of individuals captured in the same trapping period. By the end of the first trapping period, two individuals had already been recaptured within new groups (hence $2.6 \%$ recapture rate). Mean recapture rate ( $\mathrm{N}$ recaptures / $\mathrm{N}$ captures within a trapping period, excluding the first trapping period) at Kuruman was $73.1 \%$, and $54.8 \%$ at Lonely.

\begin{tabular}{|c|c|c|c|c|c|c|c|c|c|c|}
\hline Location & Trapping Period & Period Start & Period End & $\begin{array}{l}\mathbf{N} \\
\text { Groups } \\
\end{array}$ & $\begin{array}{l}\mathbf{N} \\
\text { Males }\end{array}$ & $\begin{array}{l}\mathbf{N} \\
\text { Females }\end{array}$ & $\begin{array}{l}\mathbf{N} \\
\text { Ind }\end{array}$ & $\begin{array}{l}\mathbf{N} \\
\text { New Ind }\end{array}$ & $\begin{array}{l}\text { N } \\
\text { Recap Ind }\end{array}$ & $\begin{array}{l}\text { Recap } \\
\%\end{array}$ \\
\hline Kuruman & 1 & 2013-11-17 & $2014-05-21$ & 10 & 35 & 41 & 76 & 74 & 2 & $2.6 \%$ \\
\hline Kuruman & 2 & 2014-07-27 & 2014-11-24 & 8 & 19 & 28 & 47 & 9 & 38 & $80.9 \%$ \\
\hline Kuruman & 3 & $2015-02-10$ & 2015-04-14 & 9 & 21 & 28 & 49 & 7 & 42 & $85.7 \%$ \\
\hline Kuruman & 4 & 2015-09-16 & $2015-10-22$ & 12 & 13 & 30 & 43 & 8 & 35 & $81.4 \%$ \\
\hline Kuruman & 5 & 2016-02-04 & 2016-06-06 & 17 & 20 & 38 & 58 & 15 & 43 & $74.1 \%$ \\
\hline Kuruman & 6 & 2016-07-25 & 2016-09-12 & 4 & 2 & 4 & 6 & 1 & 5 & $83.3 \%$ \\
\hline Kuruman & 7 & 2017-01-16 & 2017-05-30 & 27 & 68 & 51 & 119 & 68 & 51 & $42.9 \%$ \\
\hline Kuruman & 8 & 2017-07-31 & 2017-10-26 & 24 & 67 & 61 & 128 & 40 & 88 & $68.8 \%$ \\
\hline Kuruman & 9 & 2018-03-17 & 2018-05-30 & 24 & 79 & 70 & 149 & 46 & 103 & $69.1 \%$ \\
\hline Kuruman & 10 & 2018-09-14 & 2018-12-04 & 22 & 71 & 68 & 139 & 34 & 105 & $75.5 \%$ \\
\hline Kuruman & 11 & 2019-03-09 & 2019-04-24 & 20 & 83 & 75 & 158 & 41 & 117 & $74.1 \%$ \\
\hline Kuruman & 12 & 2019-09-09 & 2019-11-30 & 24 & 93 & 97 & 190 & 65 & 125 & $65.8 \%$ \\
\hline Kuruman & 13 & 2020-03-05 & $2020-05-27$ & 35 & 114 & 128 & 242 & 60 & 182 & $75.2 \%$ \\
\hline Lonely & 1 & 2013-09-23 & 2013-10-06 & 3 & 20 & 18 & 38 & 38 & 0 & $0.0 \%$ \\
\hline Lonely & 2 & 2014-03-01 & 2014-07-03 & 17 & 40 & 45 & 85 & 58 & 27 & $31.8 \%$ \\
\hline Lonely & 3 & 2014-09-16 & 2014-10-27 & 9 & 19 & 29 & 48 & 13 & 35 & $72.9 \%$ \\
\hline Lonely & 4 & 2015-01-29 & 2015-04-02 & 10 & 14 & 20 & 34 & 9 & 25 & $73.5 \%$ \\
\hline Lonely & 5 & 2016-02-08 & 2016-05-12 & 13 & 24 & 41 & 65 & 30 & 35 & $53.8 \%$ \\
\hline Lonely & 6 & 2016-09-09 & 2016-10-13 & 16 & 55 & 54 & 109 & 66 & 43 & $39.4 \%$ \\
\hline Lonely & 7 & 2017-05-30 & 2017-08-02 & 24 & 85 & 72 & 157 & 67 & 90 & $57.3 \%$ \\
\hline
\end{tabular}


Table S2. Summary of the analyses carried out in the paper, with information on the data used.

\begin{tabular}{|c|c|c|c|c|c|c|c|}
\hline Analysis & Data & $\begin{array}{l}\text { Total } \\
\text { rows of } \\
\text { data }\end{array}$ & $\begin{array}{c}\text { Total } \\
\text { unique } \\
\text { inds }\end{array}$ & $\begin{array}{l}\text { Total } \\
\text { unique } \\
\text { groups }\end{array}$ & $\begin{array}{l}\text { Included } \\
\text { incomplet } \\
\text { e captures }\end{array}$ & Method & Comments \\
\hline $\begin{array}{l}\text { Status-related } \\
\text { survivorship in } \\
\text { females }\end{array}$ & $\begin{array}{l}\text { All capture events from females, } \\
\text { irrespective of age (weight) at first } \\
\text { capture. Additional rows generated for } \\
\text { when individuals disappeared or were } \\
\text { right-censored. } \\
\text { "Fates_Allfemales.csv" }\end{array}$ & F: 1315 & F: 362 & F: 80 & Yes & $\begin{array}{l}\text { Multi-state Markov } \\
\text { model. }\end{array}$ & $\begin{array}{l}\text { - Right-censored individuals that were still } \\
\text { present for the last trapping window at } \\
\text { either study location. } \\
\text { - Four states: i- in-group non-breeder, ii- } \\
\text { single female, iii- breeder, iv - } \\
\text { disappeared/dead } \\
\text { - Figure 2, Table S3 }\end{array}$ \\
\hline $\begin{array}{l}\text { Growth: body } \\
\text { mass }\end{array}$ & $\begin{array}{l}\text { Body mass across successive capture } \\
\text { events (a capture and recapture) } \\
\text { "FieldMR_Growth_BodyMass.csv" }\end{array}$ & $\begin{array}{l}\text { F: } 381 \\
\text { M: } 456\end{array}$ & $\begin{array}{l}\text { F: } 193 \\
\text { M: } 214\end{array}$ & $\begin{array}{l}\text { F: } 48 \\
\text { Male: } \\
39\end{array}$ & NA & $\begin{array}{l}\text { Non-linear mixed } \\
\text { effects model with von } \\
\text { Bertalanffy interval } \\
\text { equation. } \\
\text { Males and females } \\
\text { analysed separately. }\end{array}$ & $\begin{array}{l}\text { - Removed breeding females so that } \\
\text { pregnancy weights not included. } \\
\text { - Only chose recaptures falling 100-365 } \\
\text { days after the first capture. } \\
\text { - Figure 5, Figure S9, Table S5 } \\
\text { - Follow-up analysis to check effect of } \\
\text { group size on asymptotic mass (above; } \\
\text { Figure S10) }\end{array}$ \\
\hline $\begin{array}{l}\text { Growth: } \\
\text { incisor width }\end{array}$ & $\begin{array}{l}\text { Incisor width across successive capture } \\
\text { events (a capture and recapture) } \\
\text { "FieldMR_Growth_TeethWidth.csv" }\end{array}$ & $\begin{array}{l}\text { F: } 328 \\
\text { M: } 381\end{array}$ & $\begin{array}{l}\text { F: } 180 \\
\text { M: } 198\end{array}$ & $\begin{array}{l}\text { F: } 47 \\
\text { Male: } \\
38\end{array}$ & NA & $\begin{array}{l}\text { Non-linear mixed } \\
\text { effects model with von } \\
\text { Bertalanffy interval } \\
\text { equation. } \\
\text { Males and females } \\
\text { analysed separately. } \\
\end{array}$ & $\begin{array}{l}\text { - Only chose recaptures falling 100-365 } \\
\text { days after the first capture. } \\
\text { - Figure S9, Table S7 }\end{array}$ \\
\hline $\begin{array}{l}\text { Body } \\
\text { condition of } \\
\text { females }\end{array}$ & $\begin{array}{l}\text { All body mass measurements with an } \\
\text { associated skeletal trait measurement } \\
\text { (either teeth width of body length) } \\
\text { "FieldMR_SingleFemaleCondition_Bo } \\
\text { dyLength.csv" } \\
\text { "FieldMR_SingleFemaleCondition_Te } \\
\text { ethWidth.csv" }\end{array}$ & $\begin{array}{l}\text { F } \\
\text { incisor } \\
\text { width: } \\
353 \\
\text { F body } \\
\text { length: } \\
419\end{array}$ & $\begin{array}{l}\text { F } \\
\text { incisor } \\
\text { width: } \\
177 \\
\text { F body } \\
\text { length: } \\
213\end{array}$ & $\begin{array}{l}\text { F incisor } \\
\text { width: } \\
64 \\
\text { F body } \\
\text { length: } \\
67\end{array}$ & NA & $\begin{array}{l}\text { Scaled major axis } \\
\text { regression of } \ln \text { (body } \\
\text { mass) } \sim \ln \text { (skeletal } \\
\text { trait). } \\
\text { In both cases, a separate } \\
\text { slope of intercept for }\end{array}$ & $\begin{array}{l}\text {-Included information from females that } \\
\text { were either an in-group non-breeder or a } \\
\text { single female. As the main question was } \\
\text { whether single females had a similar body } \\
\text { condition to in-group non-breeding females, } \\
\text { we only considered in-group non-breeders } \\
\text { when their skeletal trait was greater than the } \\
\text { smallest recorded value in single females }\end{array}$ \\
\hline
\end{tabular}




\begin{tabular}{|c|c|c|c|c|c|c|c|}
\hline & & & & & & $\begin{array}{l}\text { single females was not } \\
\text { supported. }\end{array}$ & $\begin{array}{l}\text { (i.e. removing young females still in their } \\
\text { natal group to ensure this didn't bias } \\
\text { allometric scaling). } \\
\text { - Figure } 3\end{array}$ \\
\hline $\begin{array}{l}\text { Within-group } \\
\text { recruitment } \\
\text { rate } \\
\text { (longitudinal) }\end{array}$ & $\begin{array}{l}\text { All complete group captures and } \\
\text { recaptures separated by } 100-365 \text { days. } \\
\text { "FieldMR_Recruitment_Longitudinal.c } \\
\text { sv" }\end{array}$ & $\begin{array}{l}78 \\
\text { capture- } \\
\text { recaptur } \\
\text { e events }\end{array}$ & NA & $\begin{array}{l}33 \\
\text { groups }\end{array}$ & No & $\begin{array}{l}\text { Generalised linear } \\
\text { mixed effects model } \\
\text { with Poisson error } \\
\text { distribution. } \\
\text { Included offset term for } \\
\text { time interval. }\end{array}$ & $\begin{array}{l}\text {-Within-group recruits classed as all new } \\
\text { individuals in the group on recapture that } \\
\text { were less than year of age (body mass }< \\
80 \mathrm{~g} \text { for females, body mass }<100 \mathrm{~g} \text { for } \\
\text { males")" } \\
\text { - Only included groups if capture history } \\
\text { indicated the presence of a breeding female. } \\
\text { - Figure 4; Table S4 }\end{array}$ \\
\hline $\begin{array}{l}\text { Within-group } \\
\text { recruitment } \\
\text { rate } \\
\text { (experimental) }\end{array}$ & $\begin{array}{l}\text { The number of new recruits on the first } \\
\text { recapture of experimentally created } \\
\text { pairs, which were compared to the } \\
\text { number of recruits in established } \\
\text { groups captured across the same time } \\
\text { period. } \\
\text { "FieldMR_Recruitment_Experimental. } \\
\text { csv" }\end{array}$ & $\begin{array}{l}\text { Pairings } \\
: 8 \\
\text { Time- } \\
\text { matched } \\
\text { groups: } \\
9\end{array}$ & NA & NA & No & t-test on recruitment rate & - Figure 4 \\
\hline
\end{tabular}


891

896

904

Table S3. Status-related survivorship. Probability of transitioning between states over a oneyear and a two-year period. Transition probabilities estimated from a multi-state model fitted to all females that were captured across the duration of the study. $n=362$ females. Estimates related to survival probability are filled in grey. Confidence intervals estimated using the delta method.

\begin{tabular}{|c|c|c|}
\hline Transition & $\begin{array}{l}1 \text { year probability } \\
(95 \% \mathrm{CI})\end{array}$ & $\begin{array}{l}2 \text { year probability } \\
(95 \% \mathrm{CI})\end{array}$ \\
\hline $\begin{array}{l}\text { in-group non-breeder } \rightarrow \text { in-group non- } \\
\text { breeder }\end{array}$ & $0.525(0.466,0.568)$ & $0.276(0.221,0.323)$ \\
\hline in-group non-breeder $\rightarrow$ single female & $0.069(0.0$ & $0.078(0$. \\
\hline in-group non-breeder $\rightarrow$ breeder & 077) & $115)$ \\
\hline in-group non-breeder $\rightarrow$ dead/dis & 0.358 & $.624)$ \\
\hline single female $\rightarrow$ single female & $0.612(0.469,0.716)$ & $0.375(0.217,0.506)$ \\
\hline$e \rightarrow b$ & 0.18 & 404) \\
\hline single female $\rightarrow$ dead/disappeared & $0.207(0.131,0.318)$ & $0.358(0.249,0.512)$ \\
\hline breeder $\rightarrow$ single female & 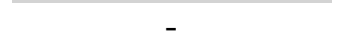 & \\
\hline breeder $\rightarrow$ bre & 0.86 & $(0.60$ \\
\hline breeder $\rightarrow$ dead/disappeared & $0.133(0.079,0.223)$ & $0.248(0.151,0.400)$ \\
\hline
\end{tabular}

Table S4. The recruitment rate of offspring into groups in Damaraland mole-rats in the wild. Recruitment was modelled with a generalised linear model with negative binomial error distribution. Models were fitted to $n=77$ group-level recapture events in 33 distinct breeding groups. All estimates are provided on the link scale (log-link), and continuous variables are z-score transformed. Models also included an offset for the $\log$ (time between capture and recapture).

\begin{tabular}{lcccc}
\hline & $\begin{array}{c}\text { Mean } \\
\text { Estimate }\end{array}$ & $\begin{array}{c}\text { Std. } \\
\text { Error. }\end{array}$ & z-value & p-value \\
\hline Fixed effects & & & & \\
Intercept & -4.538 & 0.108 & & \\
Group Size & 0.216 & 0.106 & 2.04 & $\mathbf{0 . 0 4 2}$ \\
Breeder mass & -0.090 & 0.089 & -1.02 & 0.309 \\
Rainfall & 0.159 & 0.086 & 1.85 & 0.065 \\
& & & & \\
Random effect & Variance & Std Dev & & \\
Group ID $(\mathrm{n}=33)$ & 0.129 & 0.359 & & \\
\hline
\end{tabular}


906 Table S5. Body mass growth of Damaraland mole-rats in the wild. Model summaries 907 provide estimates from the non-linear mixed effects that incorporated group size into von 908 Bertalanffy interval equations. Models were fitted to $n=381$ recapture events in females, and $909 \mathrm{n}=456$ recapture events in males. Estimates provide the population mean for the fixed effects, 910 the standard deviation for the random effects.

911

\begin{tabular}{lcccc}
\hline Females & $\begin{array}{c}\text { Mean } \\
\text { Estimate }\end{array}$ & $\begin{array}{c}\text { Std. } \\
\text { Error. }\end{array}$ & t-value, df & p-value \\
\hline Fixed effects & 118.26 & 2.01 & $58.72,185$ & $<\mathbf{0 . 0 0 1}$ \\
$A$ & -9.42 & 1.47 & $6.41,185$ & $<\mathbf{0 . 0 0 1}$ \\
$A_{G S}$ & 0.00447 & 0.00003 & $16.02,185$ & $<\mathbf{0 . 0 0 1}$ \\
$K$ & 0.00155 & 0.00032 & $4.90,185$ & $<\mathbf{0 . 0 0 1}$ \\
$k_{G S}$ & & & & \\
Random effect & Variance & Residual & & \\
$A \sim 1 \mid$ AnimalID & 15.79 & 9.18 & & \\
$k \sim 1 \mid$ AnimalID & Negligible & & & \\
& & & & \\
Males & Mean & Std. & t-value, df & p-value \\
& Estimate & Error. & & \\
Fixed effects & & & & \\
$A$ & 149.70 & 2.84 & $52.74,239$ & $<\mathbf{0 . 0 0 1}$ \\
$A_{G S}$ & -8.10 & 2.02 & $4.00,239$ & $<\mathbf{0 . 0 0 1}$ \\
$k$ & 0.00368 & 0.00002 & $17.90,239$ & $<\mathbf{0 . 0 0 1}$ \\
$k_{G S}$ & 0.00084 & 0.00020 & $4.36,239$ & $\mathbf{0 . 0 0 1}$ \\
Random effect & Variance & Residual & & \\
$A \sim 1 \mid$ AnimalID & 21.47 & 12.51 & & \\
$k \sim 1 \mid$ AnimalID & Negligible & & & \\
\hline
\end{tabular}


913 Table S6. The adult body mass of mole-rat non-breeders. Female and male mass was 914 modelled using a linear mixed effects model with Gaussian error distribution. In either case, 915 models were fitted to non-breeding individuals that were captured at least one year after they 916 were first captured at less than 1 year of age $(<100 \mathrm{~g}$ for males, $<80 \mathrm{~g}$ for females $)$. They 917 therefore represent the average body mass of all non-breeding individuals captured beyond 1 918 year of age, with most data coming from individuals far older than this. Group size categories 919 were separated at the tertiles within each data set; for males at 1-9 (small), 10-16 (medium), $920>16$ (large); for females at 1-7 (small), 8-15 (medium), >15 (large). $\mathrm{n}=65$ unique females from 92125 groups. $\mathrm{n}=73$ unique males from 23 groups. See Figure S10.

922

\begin{tabular}{|c|c|c|c|c|c|}
\hline Females $(n=111)$ & $\begin{array}{c}\text { Mean } \\
\text { Estimate }\end{array}$ & $\begin{array}{c}\text { Std. } \\
\text { Error. }\end{array}$ & $\begin{array}{l}\text { t-value, } \\
\text { df }\end{array}$ & Chisq, df & p-value \\
\hline \multicolumn{6}{|l|}{ Fixed effects } \\
\hline Intercept & 112.95 & 4.41 & & & \\
\hline $\begin{array}{l}\text { Adult group size: } \\
\text { medium }\end{array}$ & -4.12 & 4.62 & -0.892 & $1.23,2$ & 0.54 \\
\hline Adult group size: large & 1.15 & 6.27 & 0.183 & & \\
\hline Yearly quarter: second & -4.55 & 3.87 & -1.178 & $1.90,3$ & 0.59 \\
\hline Yearly quarter: third & -4.15 & 4.11 & -1.009 & & \\
\hline Yearly quarter: fourth & -2.06 & 3.97 & -0.511 & & \\
\hline Early life group size & 3.08 & 2.62 & -1.176 & $1.23,1$ & 0.27 \\
\hline Random effect & Variance & Std Dev & & & \\
\hline Animal ID:Group ID & 162.2 & 12.73 & & & \\
\hline Animal ID & 114.6 & 10.71 & & & \\
\hline Residual & 128.5 & 11.34 & & & \\
\hline Males $(n=152)$ & $\begin{array}{l}\text { Mean } \\
\text { Estimate }\end{array}$ & $\begin{array}{l}\text { Std. } \\
\text { Error. }\end{array}$ & $\begin{array}{l}\text { t-value, } \\
\text { df }\end{array}$ & & \\
\hline \multicolumn{6}{|l|}{ Fixed effects } \\
\hline Intercept & 151.30 & 5.44 & & & \\
\hline $\begin{array}{l}\text { Adult group size: } \\
\text { medium }\end{array}$ & -6.39 & 4.49 & -1.423 & $2.13,2$ & 0.34 \\
\hline Adult group size: large & -6.15 & 5.36 & -1.148 & & \\
\hline Yearly quarter: second & -14.22 & 3.36 & -4.238 & $27.15,3$ & $<0.001$ \\
\hline Yearly quarter: third & -15.53 & 3.24 & 4.789 & & \\
\hline Yearly quarter: fourth & -12.03 & 3.57 & -3.373 & & \\
\hline Early life group size & -6.56 & 3.33 & -1.967 & $2.99,1$ & 0.084 \\
\hline Random effect & Variance & Std Dev & & & \\
\hline Animal ID:Group ID & 493.2 & 22.21 & & & \\
\hline Animal ID & 159.4 & 12.62 & & & \\
\hline Residual & 141.0 & 11.88 & & & \\
\hline
\end{tabular}


924 Table S7. Incisor width growth of Damaraland mole-rats in the wild. Model summaries 925 provide estimates from the non-linear mixed effects that incorporated group size into von 926 Bertalanffy interval equations. Models were fitted to $n=328$ recapture events in females, and $927 \mathrm{n}=381$ recapture events in males. Estimates provide the population mean for the fixed effects, 928 the standard deviation for the random effects.

929

\begin{tabular}{|c|c|c|c|c|}
\hline Females & $\begin{array}{c}\text { Mean } \\
\text { Estimate }\end{array}$ & $\begin{array}{l}\text { Std. } \\
\text { Error. }\end{array}$ & t-value, df & p-value \\
\hline \multicolumn{5}{|l|}{ Fixed effects } \\
\hline$A$ & 5.75 & 0.04 & $147.60,145$ & $<0.001$ \\
\hline$A_{G S}$ & -0.11 & 0.04 & $4.17,145$ & $<0.001$ \\
\hline$K$ & 0.0053 & 0.0002 & $26.23,145$ & $<0.001$ \\
\hline$k_{G S}$ & 0.0031 & 0.0002 & $1.37,145$ & 0.17 \\
\hline Random effect & Variance & Residual & & \\
\hline$A \sim 1 \mid$ AnimalID & 0.39 & 0.16 & & \\
\hline$k \sim 1 \mid$ AnimalID & Negligible & & & \\
\hline Males & $\begin{array}{l}\text { Mean } \\
\text { Estimate }\end{array}$ & $\begin{array}{l}\text { Std. } \\
\text { Error. }\end{array}$ & t-value, df & p-value \\
\hline \multicolumn{5}{|l|}{ Fixed effects } \\
\hline$A$ & 6.48 & 0.05 & $132.11,180$ & $<0.001$ \\
\hline$A_{G S}$ & -0.05 & 0.04 & $1.32,180$ & 0.19 \\
\hline$k$ & 0.00429 & 0.0001 & $31.19,180$ & $<0.001$ \\
\hline$k_{G S}$ & 0.00007 & 0.0001 & $0.48,180$ & 0.64 \\
\hline Random effect & Variance & Residual & & \\
\hline$A \sim 1 \mid$ AnimalID & 0.47 & 0.20 & & \\
\hline$k \sim 1 \mid$ AnimalID & Negligible & & & \\
\hline
\end{tabular}

